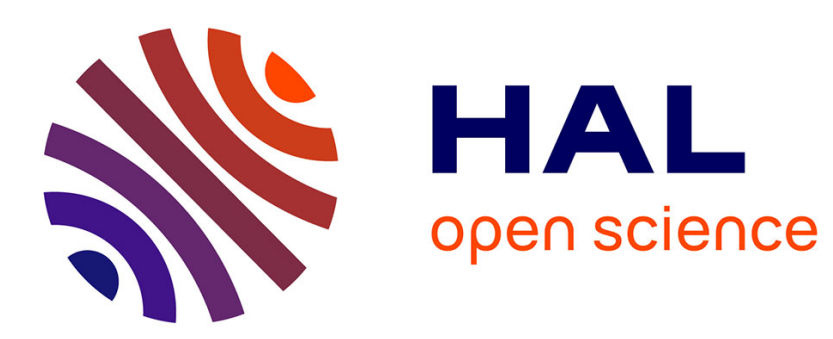

\title{
Qualitative and Numerical Analysis of a Spectral Problem with Perimeter Constraint
}

\author{
Beniamin Bogosel, Edouard Oudet
}

\section{To cite this version:}

Beniamin Bogosel, Edouard Oudet. Qualitative and Numerical Analysis of a Spectral Problem with Perimeter Constraint. SIAM Journal on Control and Optimization, 2016, 54 (1), pp.317-340. 10.1137/140999530 . hal-02009510

HAL Id: hal-02009510 https://hal.univ-grenoble-alpes.fr/hal-02009510

Submitted on 20 Feb 2019

HAL is a multi-disciplinary open access archive for the deposit and dissemination of scientific research documents, whether they are published or not. The documents may come from teaching and research institutions in France or abroad, or from public or private research centers.
L'archive ouverte pluridisciplinaire HAL, est destinée au dépôt et à la diffusion de documents scientifiques de niveau recherche, publiés ou non, émanant des établissements d'enseignement et de recherche français ou étrangers, des laboratoires publics ou privés. 


\title{
QUALITATIVE AND NUMERICAL ANALYSIS OF A SPECTRAL PROBLEM WITH PERIMETER CONSTRAINT
}

\author{
BENIAMIN BOGOSEL, ÉDOUARD OUDET
}

\begin{abstract}
We consider the problem of optimizing the $k^{\text {th }}$ eigenvalue of the Dirichlet Laplace operator under perimeter constraint. We provide a new method based on a $\Gamma$ convergence result for approximating the corresponding optimal shapes. We also give new optimality conditions in the case of multiple eigenvalues. We deduce from previous conditions the fact that optimal shapes never contain flat parts in their boundaries.
\end{abstract}

\section{INTRODUCTION}

Many works adress the shape optimization problem

$$
\min \left\{\lambda_{k}(\Omega): \Omega \subset \mathbb{R}^{d},|\Omega|=1\right\},
$$

where $\lambda_{k}$ is the $k^{\text {th }}$ eigenvalue of the Dirichlet Laplacian on $\Omega$. Faber and Krahn proved that for $k=1$ the minimizer is a ball of unit volume and Polya and Szego proved that for $k=2$ the minimizer consists of two balls of volume one half. For the case $k \geq 3$ the shapes of the minimizers are unknown. Numerical studies of the optimal shapes were performed, initially by É. Oudet in [25], and more recently improved by P. Antunes, P. Freitas [3].

In recent articles [12, 15] authors switched from the measure constraint to a perimeter constraint:

$$
\min \left\{\lambda_{k}(\Omega): \Omega \subset \mathbb{R}^{d}, \operatorname{Per}(\Omega)=1\right\} .
$$

It is not difficult to see that problem (1.2) is equivalent to

$$
\min \left\{\lambda_{k}(\Omega)+\operatorname{Per}(\Omega): \Omega \subset \mathbb{R}^{d}\right\}
$$

in the sense that any solution of (1.2) is homothetic to a solution of (1.3) and conversely. We will use freely these two equivalent formulations.

In the case $k=1$, the solution to problem (1.2) is obviously a ball as a consequence of the isoperimetric inequality and the Faber-Krahn inequality. The case $k=2, d=2$ was considered by D. Bucur, G. Buttazzo and A. Henrot in [12. The authors provided existence, regularity, qualitative and numerical results. Recently G. De Philippis and B. Velichkov [15] proved that the shape optimization problem (1.2) has a solution for any $k \in \mathbb{N}^{*}$ and for any dimension $d$. They also proved that the solution is bounded, connected, open with boundary of class $C^{1, \alpha}$ outside a closed set of Hausdorff dimension $d-8$. We underline the fact that it is not necessary to consider sets $\Omega$ contained in a bounding box $D \subset \mathbb{R}^{d}$ in order to have the existence result. Furthermore, in [15] it is proved that the optimizer found in the absence of a box constraint is bounded. Therefore, if the bounding box $D$ is large enough, the optimizer is the same as in the general case $\Omega \subset \mathbb{R}^{d}$.

The numerical studies performed by É. Oudet [25] and P. Antunes, P. Freitas [3] for problem (1.1) show that the expected minimizers do not have an obvious geometric structure for $k \geq 5$. In [12] it is proved that the optimal shape for (1.2) in the case $k=2, d=2$ does not contain any segment or any arc of circle in its boundary. This suggests that we cannot hope to find a simple geometric description of the solution of (1.2) even in the case of $k=2$.

In this context it is relevant to introduce new numerical approaches which provide a precise description of optimal candidates in two and three dimensions. One numerical approach which has been successfully used in the last few years is the following Fourier 
parametric method. Considering the formulation (1.3) we note that the monotonicity of $\lambda_{k}$ and the fact that in $\mathbb{R}^{2}$ convexification decreases perimeter imply that every solution of the problem (1.2) in the plane is convex. Thus we can represent any optimal candidate in the plane using its radial function $r(\theta)$. Furthermore, we can approximate the radial function $r$ by its truncated Fourier series $r_{n}$ ( $n$ sine and cosine coefficients). Doing this truncation, we don't perturb the eigenvalues too much. B. Osting gives an estimate of this error in 23. In this way we can represent a good approximation of the boundary of a star convex shape by a finite number of parameters. It is possible to find the partial derivatives of $\lambda_{k}\left(\Omega_{r_{n}}\right)$ with respect to the Fourier coefficients. Then a gradient descent algorithm can be used in order to find the optimal shape candidate in terms of first $2 n+1$ Fourier coefficients. This method is very precise and gives reliable estimates of computed eigenvalues. The same method is used in [3]. The method can be generalized to the three dimensional case and P. Antunes and P. Freitas announced a result in this direction [4]. A possible drawback of using this method in three or more dimensions is the fact that we do not know apriori that the solutions of (1.2) are star-convex in dimension greater than two.

A different approach consists of representing the shape $\Omega$ as a density function $\varphi$ : $D \rightarrow[0,1]$ (where $D$ is a bounded, open set of $\mathbb{R}^{2}$ ). In recent works of É. Oudet [26] and B. Bourdin, D. Bucur, É. Oudet [8], some $\Gamma$-convergence results are used in order to approximate the perimeter of $\Omega$ and the eigenvalue $\lambda_{k}(\Omega)$ by relaxed functionals calculated on a density approximation of $\Omega$. As stated above, choosing a large enough bounding box $D$, does not modify the optimizer. In the case of the $\Gamma$-convergence approximation presented in Section 3, considering a bounding box $D$ simplifies the proofs.

The first main contribution of this article is to prove that we can combine the two results above in order to produce a relaxation by $\Gamma$-convergence of $\lambda_{k}(\Omega)+\operatorname{Per}(\Omega)$. We implement this method for $d=2$ and $d=3$ and we obtain comparable results with the Fourier parametrization approach, in the two dimensional case. The advantage of our method is the fact that we do not make any topological assumptions on the optimal shape. Moreover, the numerical implementation in dimension three or greater is very similar to the one in dimension two.

Our second contribution is to provide new optimality conditions (Corollary 5.4) for this spectral shape optimization problem which are also relevant in a non differentiable context. As a matter of fact, the difficulty that arises very often in problem (1.2) is the fact that the cost function is not differentiable anymore when the optimizer does not have a simple $k^{\text {th }}$ eigenvalue. This fact was observed in our computations presented in Section 4 . Notice that the question of finding the multiplicity at the optimum is still open even for problem (1.1). Thanks to these new optimality conditions, we are able to generalize the qualitative results obtained in [12] in our general setting: for every $k$ and any $d$, the optimal shape does not contain flat parts in its boundary. The optimality condition is obtained under the hypothesis that $\Omega$ is of class $C^{3}$, which is stronger than the result proved in [15]. The optimality relation allows us to use a bootstrap argument, similar to the one used in [12], in order to prove that if $\Omega$ is of class $C^{3}$, then $\Omega$ is of class $C^{\infty}$. Thus, in order to completely solve the regularity issue for problem [1.2, it only remains to fill the gap between $C^{1, \alpha}$ and $C^{3}$.

\section{Preliminaries}

In the proof of our results we will need different theoretical tools, which are recalled below.

2.1. Spectrum of a measurable set. For well posedness reasons, it is convenient to extend the notion of Sobolev space to any measurable set $\Omega \subset \mathbb{R}^{d}$ by defining

$$
\widetilde{H}_{0}^{1}(\Omega)=\left\{u \in H^{1}\left(\mathbb{R}^{d}\right): u=0 \text { a.e. on } \Omega^{c}\right\} .
$$


In general we have $H_{0}^{1}(\Omega) \subset \widetilde{H}_{0}^{1}(\Omega)$ and we have equality if, for instance, $\Omega$ has Lipschitz boundary. Furthermore, it is proved in [19, Chapter 4] that there exists a quasi-open set $\omega \subset \Omega$ such that $\widetilde{H}_{0}^{1}(\Omega)=H_{0}^{1}(\omega)$. More technical details about the choice of this space, and why is it suitable in the study of problem (1.2), can be found in [12] and [15].

For any $\Omega \subset \mathbb{R}^{d}$ of finite measure and any $f \in L^{2}\left(\mathbb{R}^{d}\right)$ we define $R_{\Omega}(f) \in \widetilde{H}_{0}^{1}(\Omega)$ as the weak solution in $\widetilde{H}_{0}^{1}(\Omega)$ of the equation

$$
-\Delta u=f, u \in \widetilde{H}_{0}^{1}(\Omega)
$$

or equivalently as the unique minimizer in $\widetilde{H}_{0}^{1}(\Omega)$ of

$$
u \mapsto \frac{1}{2} \int_{\Omega}|\nabla u|^{2}-\int_{\Omega} f u
$$

Then $R_{\Omega}$ is a positive, self-adjoint and compact operator. As a consequence, its spectrum is discrete and its eigenvalues form a sequence converging to zero. Thus we can set

$$
\lambda_{k}(\Omega)=\frac{1}{\Lambda_{k}\left(R_{\Omega}\right)}
$$

where $0<\ldots \leq \Lambda_{k}(\Omega) \leq \ldots \leq \Lambda_{1}(\Omega)$ are the eigenvalues of $R_{\Omega}$.

If $\mu$ is a capacitary measure (i.e. $\mu(A)=0$ if $\operatorname{cap}(A)=0$ ) then $\lambda_{k}(\mu)$ is defined as the $k^{\text {th }}$ eigenvalue of the operator $-\Delta+\mu I$. The corresponding Rayleigh formulas are

$$
\lambda_{n}(\mu)=\min _{E \in S_{n}} \max _{u \in E \backslash\{0\}} \frac{\int_{D}|\nabla u|^{2} d x+\int_{D} u^{2} d \mu}{\int_{D} u^{2} d x},
$$

where the minimum is taken over $n$ dimensional subspaces of $H_{0}^{1}(D) \cap L^{2}(D ; \mu)$. Using this formula we immediately deduce the following monotonicity property: if $\mu \leq \nu$ then $\lambda_{k}(\mu) \leq \lambda_{k}(\nu)$. We note that the eigenvalues of a shape $\Omega$ correspond to the eigenvalues of the measure $+\infty \Omega^{c}$.

The notion which is well suited to the study of the convergence of Dirichlet eigenvalues is the $\gamma$-convergence. If $\left(\mu_{n}\right), \mu$ are capacitary measures we say that $\mu_{n} \gamma$-converges to $\mu$ if

$$
\left|R_{\mu_{n}}-R_{\mu}\right|_{\mathcal{L}\left(L^{2}(D)\right)} \rightarrow 0 .
$$

We have denoted $R_{\mu}$ the resolvent of the operator $-\Delta+\mu I$. In particular, if $\mu_{n} \gamma$-converges to $\mu$, then

$$
\lambda_{k}\left(\mu_{n}\right) \rightarrow \lambda_{k}(\mu)
$$

A useful characterization of the $\gamma$-convergence of a sequence of sets $\left(\Omega_{n}\right)$ to another set $\Omega$ is the Mosco convergence of the spaces $H_{0}^{1}\left(\Omega_{n}\right)$ to $H_{0}^{1}(\Omega)$. We suppose that $\Omega_{n}, \Omega$ are contained in a bounded open set $D$. We say that $H_{0}^{1}\left(\Omega_{n}\right)$ converges to $H_{0}^{1}(\Omega)$ in the sense of Mosco if the two following conditions are satisfied:

(M1) For all $u \in H_{0}^{1}(\Omega)$ there exists a sequence $u_{n} \in H_{0}^{1}\left(\Omega_{n}\right)$ such that $u_{n}$ converges strongly in $H_{0}^{1}(D)$ to $u$.

(M2) For every sequence $u_{n_{k}} \in H_{0}^{1}\left(\Omega_{n_{k}}\right)$ weakly convergent in $H_{0}^{1}(D)$ to a function $u$ we have $u \in H_{0}^{1}(\Omega)$.

For more details we refer to [11, Chapter 6] and [19].

For every measurable set $\Omega$ of finite measure we denote $w_{\Omega}$ the weak solution of the equation

$$
-\Delta w_{\Omega}=1, w_{\Omega} \in \widetilde{H}_{0}^{1}(\Omega) .
$$

We have $w_{U} \leq w_{\Omega}$ whenever $U \subset \Omega$ and

$$
H_{0}^{1}\left(\left\{w_{\Omega}>0\right\}\right)=\widetilde{H}_{0}^{1}\left(\left\{w_{\Omega}>0\right\}\right)=\widetilde{H}_{0}^{1}(\Omega) .
$$

We refer to [12], [15] for further details. 
2.2. $\Gamma$-convergence and Modica Mortola Theorem. In shape optimization, many numerical methods replace the shape variable by some unknown function. One main difficulty in our context is to associate to this kind of functional framework a way to compute the perimeter of the set. To achieve this goal, the characteristic function $\chi_{\Omega}$ will be approximated by a regular function $u \in H^{1}(\Omega)$ and the perimeter of $\Omega$ will be replaced by some smooth functional. This smooth functional is chosen from a sequence of functionals which $\Gamma$-converges to the perimeter.

The notion of $\Gamma$-convergence, introduced by de Giorgi, is a suitable tool for the study of the convergence of variational problems. For the sake of completeness, we present its definition and some of its main properties.

Definition 2.1. Let $X$ be a metric space and $F_{\varepsilon}, F: X \rightarrow[0,+\infty]$ a sequence of functionals on $X$ (defined for $\varepsilon>0$ ). We say that $F_{\varepsilon} \Gamma$-converges to $F$ and we denote $F_{\varepsilon} \stackrel{\Gamma}{\longrightarrow} F$ if the following two properties hold:

(LI) For every $x \in X$ and every $\left(x_{\varepsilon}\right) \subset X$ with $x_{\varepsilon} \rightarrow x$ we have

$$
F(x) \leq \liminf _{\varepsilon \rightarrow 0} F_{\varepsilon}\left(x_{\varepsilon}\right)
$$

(LS) For every $x \in X$ there exists $\left(x_{\varepsilon}\right) \subset X$ such that $\left(x_{\varepsilon}\right) \rightarrow x$ and

$$
F(x) \geq \limsup _{\varepsilon \rightarrow 0} F_{\varepsilon}\left(x_{\varepsilon}\right) .
$$

Given $x_{0} \in X$ we will call recovery sequence a sequence $\left(x_{\varepsilon}\right)$, which satisfies property (2.2). This sequence satisfies, in particular, the relation

$$
\lim _{\varepsilon \rightarrow 0} F_{\varepsilon}\left(x_{\varepsilon}\right)=F(x) .
$$

Here are three main properties of the $\Gamma$-convergence.

Proposition 2.2. If $F_{\varepsilon} \stackrel{\Gamma}{\longrightarrow} F$ in $X$ then the following properties hold:

(i) $F$ is lower semicontinuous;

(ii) If $G: X \rightarrow[0, \infty)$ is a continuous functional then

$$
F_{\varepsilon}+G \stackrel{\Gamma}{\longrightarrow} F+G \text {. }
$$

(iii) Suppose $x_{\varepsilon}$ minimizes $F_{\varepsilon}$ over $X$. Then every limit point of $\left(x_{\varepsilon}\right)$ is a minimizer for $F$.

The last property suggests that we could approximate a minimizer of $F$ by a minimizer of $F_{\varepsilon}$ for $\varepsilon$ small enough. This method was successfully used in [8, 26].

Sometimes it is difficult to prove the (LS) property (2.2) for every $x \in X$. Having an element $x$ with some good regularity properties may aid in constructing the recovery sequence. The following procedure, of reducing the class of elements $x$ for which we prove (2.2) to a dense subset of $\{F<+\infty\}$, is classical (see for example [9], [10]).

Proposition 2.3. Let $\mathcal{D} \subset\{F<+\infty\}$ be a dense subset of $X$, such that for every $x \in$ $\{F<+\infty\}$ and $\left(u_{n}\right) \subset \mathcal{D}$, with $\left(u_{n}\right) \rightarrow x$ we have

$$
\limsup _{n \rightarrow \infty} F\left(u_{n}\right) \leq F(x)
$$

Suppose that for every $x \in \mathcal{D}$, the property (2.2) is verified. Then (2.2) is verified in general.

The result stated below is due to Modica and Mortola [20, and it provides an approximation of the perimeter using $\Gamma$-convergence. 
Theorem 2.4. Let $D$ be a bounded open set and let $W: \mathbb{R} \rightarrow[0, \infty)$ be a continuous function such that $W(z)=0$ if and only if $z \in\{0,1\}$. Denote $c=2 \int_{0}^{1} \sqrt{W(s)} d s$. We define $F_{\varepsilon}, F: L^{1}(D) \rightarrow[0,+\infty]$ by

$$
F_{\varepsilon}(u)= \begin{cases}\varepsilon \int_{D}|\nabla u|^{2}+\frac{1}{\varepsilon} \int_{D} W(u) & u \in H^{1}(D) \\ +\infty & \text { otherwise }\end{cases}
$$

and

$$
F(u)= \begin{cases}c \operatorname{Per}\left(u^{-1}(1)\right) & u \in B V(D ;\{0,1\}) \\ +\infty & \text { otherwise }\end{cases}
$$

then

$$
F_{\varepsilon} \stackrel{\Gamma}{\longrightarrow} F
$$

in the $L^{1}(D)$ topology.

For a proof we refer to [1] or [13]. In the numerical simulations we fix the potential

$$
W(s)=s^{2}(1-s)^{2}
$$

which imposes the corresponding constant $c=1 / 3$.

Remark 2.5. In general if $F_{\varepsilon} \stackrel{\Gamma}{\longrightarrow} F$ and $G_{\varepsilon} \stackrel{\Gamma}{\longrightarrow} G$ we cannot conclude that $F_{\varepsilon}+G_{\varepsilon} \stackrel{\Gamma}{\longrightarrow}$ $F+G$. Thus, the result proved in Section 3 is not trivial. One sufficient condition for the above implication to hold would be that for each $u$ we could find the same recovery sequence for $F$ and $G$. For more details and examples see [9].

2.3. Perturbation theory for eigenvalues. Let $\left(f_{\varepsilon}\right)$ be a family of diffeomorphisms of $\mathbb{R}^{d}$ which depend analytically of $\varepsilon$, such that $f_{0}$ is the identity. Each such family of diffeomorphisms determines a sequence of perturbations $\left(\Omega_{\varepsilon}\right)=\left(f_{\varepsilon}(\Omega)\right)$ of $\Omega$. The vector field $V=\left.\frac{d}{d \varepsilon} f_{\varepsilon}\right|_{\varepsilon=0}$ is called the direction of the perturbation. One natural question is to see whether the map

$$
\varepsilon \mapsto \lambda_{k}\left(\Omega_{\varepsilon}\right)
$$

is differentiable at $\varepsilon=0$. It is known that the above map is differentiable if and only if $\lambda_{k}(\Omega)$ is simple. Nevertheless, it is possible to prove that if $\lambda_{k}(\Omega)$ has multiplicity $p>1$ and if we consider an analytic perturbation $\Omega_{\varepsilon}=f_{\varepsilon}(\Omega)$, then the $p$ corresponding eigenvalues move on $p$ smooth curves as $\varepsilon$ varies. The differentiability is lost because the $p$ eigenvalues change their places on the $p$ smooth curves as $\varepsilon$ passes through zero, due to their ordering. We could recover some informations on differentiability if we relabel them. This method has been used in [17]. We present below some of the results needed to derive our optimality conditions.

Consider $\Omega$ a bounded, open set of class $C^{3}$ in $\mathbb{R}^{d}$; therefore the mean curvature $\mathcal{H}$ is well defined and continuous. We denote by $n$ the outer normal to $\Omega$. Any perimeter preserving perturbation $\Omega_{\varepsilon}=f_{\varepsilon}(\Omega)$ induces a function $v=\left\langle\left.\frac{d}{d \varepsilon} f_{\varepsilon}\right|_{\varepsilon=0}, n\right\rangle$ on $\partial \Omega$ satisfying $\int_{\partial \Omega} \mathcal{H} v d \sigma=$ 0 . We denote by $\mathcal{P}_{0}(\partial \Omega)$ the set of $C^{1}$ functions on $\partial \Omega$ such that $\int_{\partial \Omega} \mathcal{H} v d \sigma=0$. We denote by $\operatorname{div}_{\Gamma}$ the tangential divergence with respect to $\Gamma$. We refer to [19, Section 5.4.3], for a precise description of $\operatorname{div}_{\Gamma}$.

Lemma 2.6. Let $v \in \mathcal{P}_{0}(\partial \Omega)$. Then there exists an analytic perimeter preserving deformation $\Omega_{\varepsilon}=f_{\varepsilon}(\Omega)$ such that $v=\left\langle\left.\frac{d}{d \varepsilon} f_{\varepsilon}\right|_{\varepsilon=0}, n\right\rangle$.

Proof: Let $U$ be an open neighborhood of $\bar{\Omega}$ and $\tilde{v}, \tilde{n}$ be $C^{1}$ extensions of $v, n$ to $U$. For $\varepsilon$ sufficiently small, the map $\varphi_{\varepsilon}(x)=x+\varepsilon \tilde{v}(x) \tilde{n}(x)$ is a diffeomorphism from $\Omega$ to $\varphi_{\varepsilon}(\Omega)$ (local inversion theorem). This deformation is analytic in $\varepsilon$, but is not necessarily perimeter-preserving. 
Let $X$ be an analytic vector field on $U$ such that $\int_{\partial \Omega} \operatorname{div}_{\partial \Omega} X \neq 0$ and let $u_{t}$ be the one parameter group of diffeomorphisms associated to $X$. Define $(t, \varepsilon) \mapsto G(t, \varepsilon)=\operatorname{Per}\left(u_{t} \circ\right.$ $\left.\varphi_{\varepsilon}(\Omega)\right)$. Using the fact that $\left.\frac{d u_{t}}{d t}\right|_{t=0}=X$ and Proposition 5.4.18 from [19] we obtain

$$
\frac{\partial G}{\partial t}(0,0)=\frac{d}{d t} \operatorname{Per}\left(u_{t}(\Omega)\right)=\int_{\partial \Omega} \operatorname{div}_{\partial \Omega} X d \sigma \neq 0 .
$$

Therefore we can apply the implicit function theorem around $(0,0)$ to see that there exists an analytic function $\varepsilon \mapsto t(\varepsilon)$ defined on a neighborhood $(-\eta, \eta)$ of 0 such that

$$
G(t(\varepsilon), \varepsilon)=G(0,0)=\operatorname{Per}(\Omega) .
$$

Thus the deformation $g_{\varepsilon}=u_{t(\varepsilon)} \circ \varphi_{\varepsilon}$ is perimeter preserving. Moreover, using Propositions 5.4.9 and 5.4.18 from [19], we have

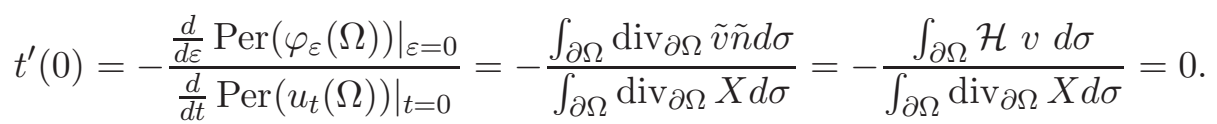

Therefore, if we set $H(t, \varepsilon)=u_{t} \circ \varphi_{\varepsilon}$ then

$$
\left.\frac{d}{d \varepsilon} g_{\varepsilon}(x)\right|_{\varepsilon=0}=\frac{d}{d t} H(t(0), 0) t^{\prime}(0)+\frac{d}{d \varepsilon} H(t(0), 0)=\left.\frac{d \varphi_{\varepsilon}}{d \varepsilon}\right|_{\varepsilon=0}=\tilde{v}(x) \tilde{n}(x)=v(x) n(x)
$$

for $x \in \partial \Omega$. In conclusion, $g_{\varepsilon}$ is the desired perturbation.

Below we present two results from [17, which will be used freely in the rest of the article. We omit the proofs, as they can be found in the cited article.

Lemma 2.7. Let $\lambda$ be an eigenvalue of multiplicity $p$ of the Dirichlet Laplacian on $\Omega$. For any analytic deformation $\Omega_{\varepsilon}$ of $\Omega$ there exist $p$ families of real numbers $\left(\Lambda_{i, \varepsilon}\right)_{i \leq p}$ and $p$ families of functions $\left(u_{i, \varepsilon}\right)_{i \leq p} \subset C^{\infty}\left(\Omega_{\varepsilon}\right)$, depending analytically on $\varepsilon$, satisfying for all $\varepsilon \in\left(-\varepsilon_{0}, \varepsilon_{0}\right)$ and for all $i \in\{1, \ldots, p\}$ :

(a) $\Lambda_{i, 0}=\lambda$.

(b) The family $\left\{u_{1, \varepsilon}, \ldots, u_{p, \varepsilon}\right\}$ is orthonormal in $L^{2}\left(\Omega_{\varepsilon}\right)$.

(c) We have $\left\{\begin{array}{l}-\Delta u_{i, \varepsilon}=\Lambda_{i, \varepsilon} u_{i, \varepsilon} \text { in } \Omega_{\varepsilon} \\ u_{i, \varepsilon}=0 \text { on } \partial \Omega_{\varepsilon} .\end{array}\right.$

Lemma 2.8. Let $\lambda$ be an eigenvalue of multiplicity $p$ of the Dirichlet Laplace operator and denote $E_{\lambda}$ the corresponding eigenspace. Let $\Omega_{\varepsilon}=f_{\varepsilon}(\Omega)$ be an analytic deformation of $\Omega$. Let $\left(\Lambda_{i, \varepsilon}\right)_{i \leq p}$ and $\left(u_{i, \varepsilon}\right)_{i \leq p}$ be like in Lemma 2.7. Then $\Lambda_{i}^{\prime}=\left.\frac{d}{d \varepsilon} \Lambda_{i, \varepsilon}\right|_{\varepsilon=0}$ are the eigenvalues of the quadratic form $q_{v}$ defined on $E_{\lambda} \subset L^{2}(\Omega)$ by

$$
q_{v}(u)=-\int_{\partial \Omega}\left(\frac{\partial u}{\partial n}\right)^{2} v d \sigma
$$

where $v=\left\langle\frac{d}{d \varepsilon} f_{\varepsilon}, n\right\rangle$. Moreover, the $L^{2}$-orthonormal basis $u_{1,0}, \ldots, u_{p, 0}$ diagonalizes $q_{v}$ on $E_{\lambda}$.

In the rest of the paper we use $\lambda_{k, \varepsilon}$ to denote $\lambda_{k}\left(\Omega_{\varepsilon}\right)$. We define the following notion of critical domain for the eigenvalues of the Dirichlet Laplacian, which generalizes the notion of local minimum or local maximum.

Definition 2.9. The domain $\Omega$ is said to be critical for the $k^{\text {th }}$ eigenvalue of the Dirichlet problem if, for any analytic perimeter-preserving deformation $\Omega_{\varepsilon}$ of $\Omega$, the right-sided and left-sided derivatives of $\lambda_{k, \varepsilon}$ (see Lemma 2.7) at $\varepsilon=0$ have opposite signs, that is

$$
\left.\frac{d}{d \varepsilon} \lambda_{k, \varepsilon}\right|_{\varepsilon=0^{+}} \times\left.\frac{d}{d \varepsilon} \lambda_{k, \varepsilon}\right|_{\varepsilon=0^{-}} \leq 0 .
$$




\section{The $\Gamma$-CONVERGENCE RESUlt}

In this section we construct a $\Gamma$-convergence approximation for $\lambda_{k}(\Omega)+\operatorname{Per}(\Omega)$. This result allows us to construct a numerical method for the study of problem (1.2), which will be presented in the next section. Consider $F: \mathbb{R}^{k} \rightarrow \mathbb{R}_{+}$a continuous function which is increasing in each variable. Let $D \subset \mathbb{R}^{d}$ be a bounded, open set. For every $\varphi: D \rightarrow \mathbb{R}_{+}$, measurable we define $\lambda_{k}(\varphi)=\lambda_{k}(\varphi d x)$, where $\varphi d x$ is seen as a capacitary measure. In the following, $q$ will be a fixed positive real parameter.

Theorem 3.1. Define $J_{\varepsilon}: L^{1}(D ;[0,1]) \rightarrow \mathbb{R}_{+} \cup\{+\infty\}$ by

$$
J_{\varepsilon}(\varphi)=F\left(\lambda_{1}\left(\frac{1-\varphi}{\varepsilon^{q}} d x\right), \ldots, \lambda_{k}\left(\frac{1-\varphi}{\varepsilon^{q}} d x\right)\right)+\varepsilon \int_{D}|\nabla \varphi|^{2}+\frac{1}{\varepsilon} \int_{D} \varphi^{2}(1-\varphi)^{2}
$$

if $\varphi \in H^{1}(D)$ and $+\infty$ otherwise. Then $J_{\varepsilon} \stackrel{\Gamma}{\longrightarrow} J$ in the $L^{1}(D)$ topology, where

$$
J(\varphi)= \begin{cases}F\left(\lambda_{1}(\Omega), \ldots, \lambda_{k}(\Omega)\right)+\frac{1}{3} \operatorname{Per}(\Omega), & \text { if } \varphi=\chi_{\Omega} \in B V(D) \\ +\infty & \text { otherwise }\end{cases}
$$

Proof: For simplicity, in the rest of the proof we denote the quantity $F\left(\lambda_{1}(\Omega), . ., \lambda_{k}(\Omega)\right)$ by $F(\Omega)$. With this notation, $F$ becomes decreasing for the inclusion, as a function of $\Omega$. We make the same convention when instead of $\Omega$ we have a measure $\mu$. Let us begin by proving the $\Gamma-$ limsup part of our result.

1. Reduction to regular domains. This part of the proof is a standard step in the proof of the $\Gamma-\lim$ sup property (see Proposition 2.3). We refer to [9, [10, for more details and examples. If $\Omega$ is regular, the construction of a recovery sequence is straightforward (see Part 2 of the proof). We are left to prove that regular sets are a dense subset $\mathcal{D}$ of $\{F<+\infty\}$ and that they satisfy the following property: for each $\Omega \in\{F<+\infty\}$ we can find $\left(\Omega_{n}\right) \subset \mathcal{D}$ such that $\chi_{\Omega_{n}} \rightarrow \chi_{\Omega}$ in $L^{1}(D)$ topology and $\limsup J\left(\chi_{\Omega_{n}}\right) \leq J\left(\chi_{\Omega}\right)$.

In [2, Thm 3.4.2 it is proved that the sets with boundary of class $C^{\infty}$ are dense in the class of finite perimeter sets, when considering the $L^{1}$ topology. Thus we can choose our dense set $\mathcal{D}$ to be the family of subsets of $D$ with finite perimeter and smooth boundary. If $\varphi$ is the characteristic function $\chi_{\Omega}$ of $\Omega$ and it belongs to $B V(D)$ then $\Omega$ is a set of finite perimeter. The theorem we cited above says that each finite perimeter $\Omega$ set can be approximated in the $L^{1}(D)$ topology with a sequence $\left(\Omega_{n}\right)$ of finite perimeter sets having smooth boundaries such that $\operatorname{Per}\left(\Omega_{n}\right) \rightarrow \operatorname{Per}(\Omega)$. At this point it is not clear if we have $\limsup F\left(\Omega_{n}\right) \leq F(\Omega)$. The objective of the following paragraphs is to construct $\left(\Omega_{n}\right)$ in

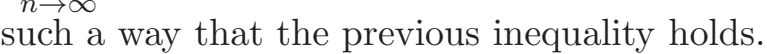

If we denote $\left(\rho_{k}\right)$ a sequence of mollifiers, we have

$$
\begin{aligned}
\operatorname{Per}(\Omega) & =\int_{\mathbb{R}^{d}}\left|D \chi_{\Omega}\right|=\lim _{k \rightarrow \infty} \int_{\mathbb{R}^{d}}\left|\nabla \chi_{\Omega} * \rho_{k}\right|= \\
& =\lim _{k \rightarrow \infty} \int_{0}^{1} \operatorname{Per}\left(\left\{\chi_{\Omega} * \rho_{k}>t\right\}\right) d t \geq \int_{0}^{1} \liminf _{k \rightarrow \infty} \operatorname{Per}\left(\left\{\chi_{\Omega} * \rho_{k}>t\right\}\right) d t
\end{aligned}
$$

where we have applied the co-area formula and Fatou's lemma. By applying Chebyshev's inequality we obtain that

$$
\left|\left\{\chi_{\Omega} * \rho_{k}>t\right\} \backslash \Omega\right|=\left|\left\{\chi_{\Omega} * \rho_{k}-\chi_{\Omega} \geq t\right\}\right| \leq \frac{1}{t} \int_{\mathbb{R}^{d}}\left|\chi_{\Omega} * \rho_{k}-\chi_{\Omega}\right|
$$

and

$$
\left|\Omega \backslash\left\{\chi_{\Omega} * \rho_{k}>t\right\}\right|=\left|\left\{\chi_{\Omega}-\chi_{\Omega} * \rho_{k} \geq 1-t\right\}\right| \leq \frac{1}{1-t} \int_{\mathbb{R}^{d}}\left|\chi_{\Omega} * \rho_{k}-\chi_{\Omega}\right| .
$$


Therefore $\chi_{\left\{\chi_{\Omega} * \rho_{k}>t\right\}}$ converges to $\chi_{\Omega}$ in the $L^{1}(D)$ topology for almost every $t \in(0,1)$. By the lower semicontinuity of the perimeter we deduce that

$$
\liminf _{k \rightarrow \infty} \operatorname{Per}\left(\left\{\chi_{\Omega} * \rho_{k}>t\right\}\right) \geq \operatorname{Per}(\Omega) .
$$

Combining this with (3.1) we obtain

$$
\liminf _{k \rightarrow \infty} \operatorname{Per}\left(\left\{\chi_{\Omega} * \rho_{k}>t\right\}\right)=\operatorname{Per}(\Omega) .
$$

for almost every $t \in(0,1)$. Sard's theorem tells us that the level sets of $\chi_{\Omega} * \rho_{k}$ are smooth for almost every $t$. Moreover, Lemma 2.95 from 2 tells us that almost all level sets of $\chi_{\Omega} * \rho_{k}$ are transversal, i.e. $\mathcal{H}^{n-1}\left(\partial\left\{\chi_{\Omega} * \rho_{k}\right\} \cap \partial D\right)=0$. In this way, we can choose the smooth, transversal approximating sets at almost every level $t \in(0,1)$.

Denote $w=R_{\Omega}(1)=R_{\omega}(1)$ where $\omega \subset \Omega$ is a quasi open set with the property that $H_{0}^{1}(\omega)=\widetilde{H}_{0}^{1}(\Omega)$. We can assume that $\|w\|_{\infty} \leq 1$ (or otherwise rescale it) so that we get $w \leq \chi_{\Omega}$ which implies that $w * \rho_{k} \leq \chi_{\Omega} * \rho_{k}$ and as a consequence $\left\{w * \rho_{k}>t\right\} \subseteq\left\{\chi_{\Omega} * \rho_{k}>t\right\}$.

We want to prove that $\limsup _{k \rightarrow \infty} F\left(\left\{w * \rho_{k}>t\right\}\right) \leq F(\{w>t\})$. Denote $A_{k}=\left\{w * \rho_{k}>\right.$ $t\} \cap\{w>t\}$. It is enough to prove that $\left(A_{k}\right) \gamma$-converges to $\{w>t\}$. Indeed, if this holds, then

$$
\limsup _{k \rightarrow \infty} F\left(\left\{w * \rho_{k}>t\right\}\right) \leq \lim _{k \rightarrow \infty} F\left(A_{k}\right)=F(\{w>t\})
$$

To prove this $\gamma$-convergence result it suffices to prove the first Mosco condition, since the second one comes from $A_{k} \subset\{w>t\}$. For more details we refer to [11, Section 4.5]. To prove the first Mosco condition it is enough to prove it on a dense subset of $H_{0}^{1}(\{w>t\})$. One such dense subset is given in [14] Prop 5.5 and is $\left\{C_{c}^{\infty}\left(\mathbb{R}^{d}\right) \cdot(w-t)^{+}\right\}$. Let $\varphi \in C_{c}^{\infty}\left(\mathbb{R}^{d}\right)$. Then if $\varphi_{k}=\varphi \cdot \min \left\{\left(w * \rho_{k}-t\right)^{+},(w-t)^{+}\right\}$we have $\varphi_{k} \rightarrow \varphi \cdot(w-t)^{+}$in $H_{0}^{1}(D)$ and $\varphi_{k} \in H_{0}^{1}\left(A_{k}\right)$. This concludes the proof of the fact that $A_{k} \gamma$-converges to $\{w>t\}$.

Therefore we have found a sequence

$$
B_{k}^{t}=\left\{w * \rho_{k}>t\right\} \subseteq C_{k}^{t}=\left\{\chi_{M} * \rho_{k}>t\right\}
$$

with $C_{k}^{t} \rightarrow \chi_{\Omega}$ in $L^{1}(D), \liminf _{k \rightarrow \infty} \operatorname{Per}\left(C_{k}^{t}\right)=\operatorname{Per}(\Omega)$ for almost every $t, F\left(C_{k}^{t}\right) \leq F\left(B_{k}^{t}\right)$ and

$$
\limsup _{k \rightarrow \infty} F\left(B_{k}^{t}\right) \leq F(\{w>t\}) .
$$

Thus, we can choose a diagonal sequence $E_{k}=C_{k}^{t_{k}}$ with $t_{k} \rightarrow 0$ such that $\chi_{E_{k}} \rightarrow \chi_{\Omega}$ in $L^{1}(D), \operatorname{Per}\left(E_{k}\right) \rightarrow \operatorname{Per}(\Omega)$ in order to obtain

$$
\limsup _{k \rightarrow \infty} F\left(E_{k}\right) \leq F(\{w>0\})=F(\omega)=F(\Omega) .
$$

2. Proof of the $\Gamma-\lim \sup$ part. Using the previous density result, it suffices to prove the $\Gamma$ - limsup only for characteristic functions of smooth sets with finite perimeter. Let $\varphi \in L^{1}(D ;[0,1])$ with $J(\varphi)<+\infty$. Then $\varphi$ is the characteristic function of a set $\Omega$ with finite perimeter. We assume, as mentioned above, that $\Omega$ has smooth boundary and that $\mathcal{H}^{n-1}(\partial \Omega \cap \partial D)=0$.

We take $\left(\varphi_{\varepsilon}\right) \subset H^{1}(D)$ to be a recovery sequence associated to the Modica-Mortola approximation (see Theorem 2.4). We recall that this sequence can be chosen to satisfy $\chi_{\Omega}(x)=\varphi_{\varepsilon}(x)$ for $d_{\Omega}(x) \notin[0, \varepsilon]$ (see [13]; here $d_{\Omega}$ represents the signed distance from a point to $\partial \Omega)$. We have $\varphi_{\varepsilon} \rightarrow \varphi$ in $L^{1}(D)$ and

$$
\lim _{\varepsilon \rightarrow 0}\left[\varepsilon \int_{D}\left|\nabla \varphi_{\varepsilon}\right|^{2} d x+\frac{1}{\varepsilon} \int_{D} \varphi_{\varepsilon}^{2}\left(1-\varphi_{\varepsilon}^{2}\right) d x\right]=\frac{1}{3} \operatorname{Per}(\Omega) .
$$


Since for every $x \in \Omega$ we have $\varphi_{\varepsilon}(x)=1$, we observe that $+\infty_{D \backslash \Omega} \geq \frac{1-\varphi_{\varepsilon}}{\varepsilon^{q}}$. By the monotonicity of $\lambda_{j}$ we have

$$
\lambda_{j}(\Omega)=\lambda_{j}\left(+\infty_{D \backslash \Omega}\right) \geq \lambda_{j}\left(\frac{1-\varphi_{\varepsilon}}{\varepsilon^{q}} d x\right) .
$$

Using the monotonicity of $F$ we obtain

$$
\limsup _{\varepsilon \rightarrow 0} F\left(\frac{1-\varphi_{\varepsilon}}{\varepsilon^{q}} d x\right) \leq F(\Omega) .
$$

3. Proof of the $\Gamma-\liminf$ part. Let $\varphi \in L^{1}(D ;[0,1])$ and $\left(\varphi_{\varepsilon}\right) \in L^{1}(D ;[0,1])$ such that $\varphi_{\varepsilon} \rightarrow \varphi$ in $L^{1}(D)$. We assume that $\liminf _{\varepsilon \rightarrow 0} J_{\varepsilon}\left(\varphi_{\varepsilon}\right)<+\infty$ since otherwise the result is obvious. The $\Gamma-\lim$ inf part of the Modica-Mortola theorem tells us that

$$
+\infty>\liminf _{\varepsilon \rightarrow 0} \varepsilon \int_{D}\left|\nabla \varphi_{\varepsilon}\right|^{2}+\frac{1}{\varepsilon} \int_{D} \varphi_{\varepsilon}^{2}\left(1-\varphi_{\varepsilon}\right)^{2} \geq \frac{1}{3} \int_{D}|D \varphi| .
$$

Thus $\varphi$ has is a characteristic function with bounded variation. This implies that $\Omega=$ $\varphi^{-1}(1)$ is a set of finite perimeter relative to $D$, and

$$
\liminf _{\varepsilon \rightarrow 0} \varepsilon \int_{D}\left|\nabla \varphi_{\varepsilon}\right|^{2}+\frac{1}{\varepsilon} \int_{D} \varphi_{\varepsilon}^{2}\left(1-\varphi_{\varepsilon}\right)^{2} \geq \frac{1}{3} \operatorname{Per}(\Omega)
$$

It remains to prove that

$$
\liminf _{\varepsilon \rightarrow 0} F\left(\frac{1-\varphi_{\varepsilon}}{\varepsilon^{q}} d x\right) \geq F(\Omega) .
$$

Since $F$ is increasing in each variable, it is enough to prove that

$$
\liminf _{\varepsilon \rightarrow 0} \lambda_{i}\left(\frac{1-\varphi_{\varepsilon}}{\varepsilon^{q}} d x\right) \geq \lambda_{i}(\Omega) .
$$

Let $w_{\varepsilon}$ be the solution of

$$
\left\{\begin{array}{l}
-\Delta w_{\varepsilon}+\frac{1-\varphi_{\varepsilon}}{\varepsilon^{q}} w_{\varepsilon}=1 \quad \text { in } D \\
w_{\varepsilon} \in H_{0}^{1}(D) .
\end{array}\right.
$$

Without loss of generality we can replace liminf with lim by taking a sequence $\varepsilon_{k}$ which realizes the liminf. Denoting $\varphi_{k}=\varphi_{\varepsilon_{k}}$, we have to prove that

$$
\lim _{n \rightarrow \infty} \lambda_{i}\left(\frac{1-\varphi_{k}}{\varepsilon_{k}^{q}} d x\right) \geq \lambda_{i}(\Omega) .
$$

By compactness there is a subsequence of $\left(w_{n_{k}}\right)$ converging weakly in $H_{0}^{1}(D)$ to $w$. We can choose a subsequence of this sequence which converges almost everywhere to $w$. For simplicity we relabel this subsequence $\left(w_{k}\right)$. It is enough to prove the inequality for $\left(\varphi_{k}\right)$ (the corresponding functions for this new sequence $\left(w_{k}\right)$ ).

Taking $w_{k}$ as test functions in the weak form of the partial differential equation we get

$$
\int_{D} \frac{1-\varphi_{k}}{\varepsilon_{k}^{q}} w_{k}^{2}=\int_{D} w_{k}-\int_{D}\left|\nabla w_{k}\right|^{2} \leq \int_{D} w_{k} \leq \int_{D} w_{D}
$$

where $w_{D}$ is the solution of

$$
\left\{\begin{array}{l}
-\Delta w_{D}=1 \quad \text { in } D \\
w_{D} \in H_{0}^{1}(D) .
\end{array}\right.
$$

We know that

$$
\liminf _{k \rightarrow \infty} \frac{1-\varphi_{k}(x)}{\varepsilon_{k}^{q}}=+\infty
$$


for $x \in \Omega^{c}$ since $1-\varphi_{k}(x) \rightarrow 1$ a.e. on $\Omega^{c}$ and $\varepsilon_{k} \rightarrow 0^{+}$. Therefore since $w_{k} \rightarrow w$ almost everywhere, if $w(x)>0, x \notin \Omega$ and $w_{k}(x) \rightarrow w(x)$ then

$$
\liminf _{k \rightarrow \infty} \frac{1-\varphi_{k}(x)}{\varepsilon_{k}^{q}} w_{k}^{2}(x)=+\infty .
$$

Fatou's Lemma tells us that

$$
+\infty>\liminf _{k \rightarrow \infty} \int_{D} \frac{1-\varphi_{k}}{\varepsilon_{k}^{q}} w_{k}^{2} \geq \int_{D} \liminf _{k \rightarrow \infty} \frac{1-\varphi_{k}}{\varepsilon_{k}^{q}} w_{k}^{2} \geq \int_{\Omega^{c}} \liminf _{k \rightarrow \infty} \frac{1-\varphi_{k}}{\varepsilon_{k}^{q}} w_{k}^{2}
$$

This inequality and the previous remarks impliy that the set $\Omega^{c} \cap\{w>0\}$ is of measure zero, and therefore $w \in \widetilde{H}_{0}^{1}(\Omega)$. Since the $\gamma$-convergence is compact, up to a subsequence we have

$$
\mu_{\varepsilon}=\frac{1-\varphi_{k}}{\varepsilon_{k}^{q}} \stackrel{\gamma}{\rightarrow} \mu \geq+\infty \Omega^{c}
$$

As a consequence, we have

$$
\lim _{k \rightarrow \infty} \lambda_{i}\left(\frac{1-\varphi_{k}}{\varepsilon_{k}^{q}} d x\right)=\lambda_{i}(\mu) \geq \lambda_{i}(\Omega)
$$

which finishes the proof of the $\Gamma-\lim$ inf part.

\section{Numerical Study of PRoblem (1.2)}

The method we developed for studying problem (1.2) combines the $\Gamma$-convergence methods used in approximating the perimeter (used in [26]) and the eigenvalues of the Laplace operator (used in [8]). The combination of the two cited methods is made possible by the $\Gamma$-convergence result proved in the previous section. As it has been underlined, our $\Gamma$-convergence method is very flexible with respect to both the dimension and the topology of the shapes. In order to evaluate the quality of our solution we recall in subsection 4.2 the method used successfully by B. Osting 23 and P. Antunes, P. Freitas 3]. In Table 1 we illustrate that both methods give the same results in the easy context of the two dimensional case. Finally, we extend previous results in the three dimensional case, where we notice that some of the optimal shapes found seem to be non-convex. This behaviour has been conjectured in [12].

4.1. Method based on the $\Gamma$-convergence result. We relax our shape optimization problem with respect to $\Omega$ by an optimization problem of an unknown function $\varphi: D \rightarrow$ $[0,1]$. In our computations we choose $D=[0, a]^{2}$ and impose periodic boundary conditions (so that the perimeter of $\Omega$ is not influenced by the boundary of $D$ ). We consider a $N \times N$ uniform grid and we represent the function $\varphi$ by its values $\left(\varphi_{i, j}\right)_{i, j=1}^{N}$ on this grid. We approximate

$$
\varphi \mapsto \varepsilon \int_{D}|\nabla \varphi|^{2}+\frac{1}{\varepsilon} \int_{D} \varphi^{2}(1-\varphi)^{2}
$$

by using centred finite differences on the considered grid. This approximation is equivalent to considering a piecewise linear function associated to the grid values.

For the eigenvalue approximation we consider the discrete form of

$$
-\Delta u_{k}+\frac{1-\varphi}{\varepsilon^{2}} u_{k}=\lambda_{k} u_{k}
$$

In order to obtain a matrix formulation, we fix an ordering on the $N \times N$ grid. We denote by $\bar{\psi}$ the vector which contains the values on the grid of the function $\psi$ with respect to this fixed ordering. We define $A$ to be the $N^{2} \times N^{2}$ matrix associated to the discrete Laplacian on the considered grid, with respect to the fixed ordering. The discretized eigenvalue problem becomes

$$
\left[A+\frac{1-\bar{\varphi}}{\varepsilon^{2}} I\right] \bar{u}_{k}=\lambda_{k} \bar{u}_{k}
$$


We used the Matlab solver eigs to solve this matrix eigenvalue problem. The expression of the discrete gradient of our functional with respect to each component of $\bar{\varphi}$ is

$$
-\frac{1}{\varepsilon^{2}} \bar{u}_{k}^{2}
$$

We refer to [8] for more details.

We can compute the gradient of $\varphi \mapsto \varepsilon \int_{D}|\nabla \varphi|^{2}+\frac{1}{\varepsilon} \int_{D} \varphi^{2}(1-\varphi)^{2}$ with respect to a perturbation $\theta$ of $\varphi \in H^{1}(D)$ as follows:

$$
\begin{aligned}
\frac{d}{d t} & {\left[\varepsilon \int_{D}|\nabla(\varphi+t \theta)|^{2}+\frac{1}{\varepsilon} \int_{D}(\varphi+t \theta)^{2}(1-(\varphi+t \theta))^{2}\right]_{t=0}=} \\
& =2 \varepsilon \int_{D}\langle\nabla \varphi, \nabla \theta\rangle+\frac{1}{\varepsilon} \int_{D}\left(2 \varphi-6 \varphi^{2}+4 \varphi^{3}\right) \theta \\
& =\int_{D}\left[-2 \varepsilon \Delta \varphi+\frac{1}{\varepsilon}\left(2 \varphi-6 \varphi^{2}+4 \varphi^{3}\right)\right] \theta
\end{aligned}
$$

Thus the discrete gradient of $\varphi \mapsto \varepsilon \int_{D}|\nabla \varphi|^{2}+\frac{1}{\varepsilon} \int_{D} \varphi^{2}(1-\varphi)^{2}$ with respect to $\varphi$ is given by

$$
2 \varepsilon\left(4 \bar{\varphi}_{i, j}-\bar{\varphi}_{i+i, j}-\bar{\varphi}_{i-1, j}-\bar{\varphi}_{i, j+1}-\bar{\varphi}_{i, j-1}\right)+\frac{1}{\varepsilon}\left(2 \bar{\varphi}_{i, j}-6 \bar{\varphi}_{i, j}^{2}+4 \bar{\varphi}_{i, j}^{3}\right) .
$$

To obtain a solution $\varphi_{0}$ of the problem

$$
\min \left[\varepsilon_{0} \int_{D}|\nabla \varphi|^{2}+\frac{1}{\varepsilon_{0}} \int_{D} \varphi^{2}(1-\varphi)^{2}+\lambda_{k}\left(\frac{1-\varphi}{\varepsilon_{0}^{2}} d x\right)\right]
$$

we start from a random configuration with a concentration around the center of the grid. Numerical experiments have shown that starting from a totally random configuration tends to lead to a shape consisting of $k$ disks. This configuration is a local minimum, but not the global one, since we know that the optimal shape is connected [15. We think this behaviour is due to the fact that when we approximate $\Omega$ by density functions, the optimization of $\lambda_{k}$ tends to separate $\Omega$ into nodal domains. Then the perimeter, which is optimized locally, transforms those domains into disks. This observation motivates our previous initialization. For the optimization part, we used the quasi-Newton algorithm LBFGS implemented in [27], 28].

The choice of the initial parameter $\varepsilon_{0}$ is important for the algorithm to converge. $\mathrm{Nu}-$ merical experiments have shown that $\varepsilon_{0} \in\left[\frac{1}{N}, \frac{4}{N}\right]$ are suitable for obtaining the expected results. This observation is well known in the phase-field community. Note that the main $\Gamma$-convergence result holds for any exponent $q$ positive. In our computations we choose $q=2$, because we observed a good numerical behaviour with this parameter. This good behavior could be explained by a well balanced effect of the cost values for $q=2$ in the scale of our discretization.

4.2. The approach of B. Osting [23] and P. Antunes, P. Freitas 3]. In order to verify our results, we compare them with the ones obtained using the Fourier boundary parametrization method mentioned in the introduction. This method is well known, and was applied in [3],23] and [24]. We present it below for the sake of completeness.

We know that the solutions to problem (1.2) in $\mathbb{R}^{2}$ are convex shapes, so every such shape is uniquely defined by its radial function $r(\theta), \theta \in[0,2 \pi)$. B. Osting proved in [23, Prop. 3.1] that the error $\left|\lambda_{k}\left(\Omega_{r}\right)-\lambda_{k}\left(\Omega_{r_{n}}\right)\right|$ can be made arbitrarily small if we choose $n$ big enough, where $r_{n}$ is the truncation of the Fourier series representation of $r$ to $2 n+1$ coefficients:

$$
r_{n}(\theta)=a_{0}+\sum_{k=1}^{n} a_{k} \cos (k \theta)+\sum_{k=1}^{n} b_{k} \sin (k \theta) .
$$




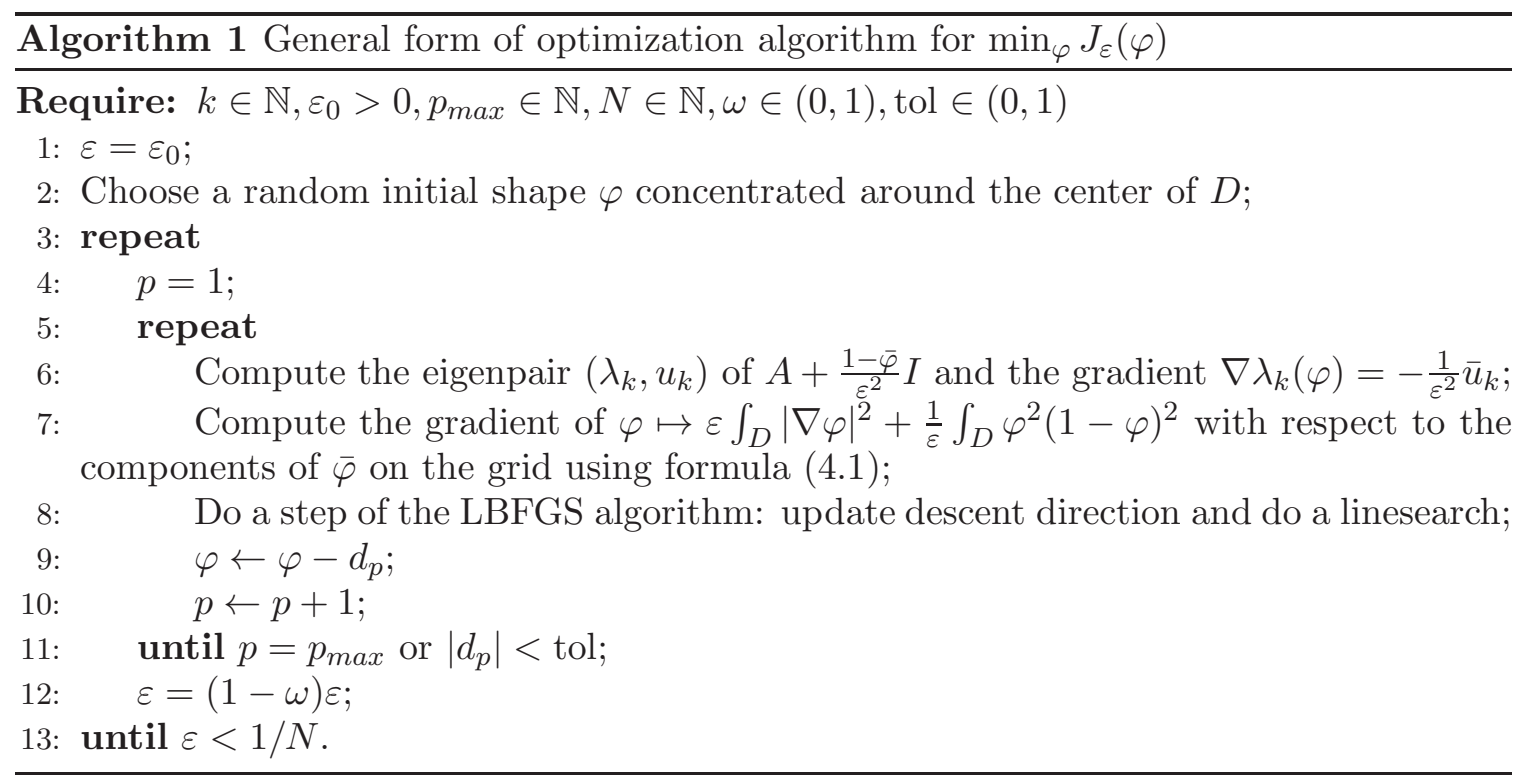

This allows us to write $\lambda_{k}(\Omega)$ as a function of $2 n+1$ variables $\lambda_{k}\left(a_{0}, a_{1}, \ldots, a_{n}, b_{1}, \ldots, b_{n}\right)$. Furthermore, using the fact that the derivative of $\lambda_{k}(\Omega)$ with respect to a perturbation $V$ of the boundary is

$$
\frac{d \lambda_{k}(\Omega)}{d V}=-\int_{\partial \Omega}\left(\frac{\partial u_{k}}{\partial n}\right)^{2}(V . n) d \sigma
$$

(proofs and other references can be found in [18, 19]) we can find that

$$
\begin{aligned}
& \frac{\partial \lambda_{k}}{\partial a_{k}}=-\int_{0}^{2 \pi} r(\theta) \cos (k \theta)\left(\frac{\partial u}{\partial n}(r(\theta), \theta)\right)^{2} d \theta \\
& \frac{\partial \lambda_{k}}{\partial b_{k}}=-\int_{0}^{2 \pi} r(\theta) \sin (k \theta)\left(\frac{\partial u}{\partial n}(r(\theta), \theta)\right)^{2} d \theta .
\end{aligned}
$$

We can find similar formulas for the derivatives of the perimeter in terms of Fourier coefficients. For computing the eigenvalues and normal derivatives of the eigenfunctions it is possible to use the publicly available software MpsPack [5].

4.3. Our numerical results. In order to solve numerically problem (1.2), in its equivalent form (1.3), we search the solutions of the relaxed problem

$$
\min \left[\varepsilon_{0} \int_{D}|\nabla \varphi|^{2}+\frac{1}{\varepsilon_{0}} \int_{D} \varphi^{2}(1-\varphi)^{2}+\lambda_{k}\left(\frac{1-\varphi}{\varepsilon_{0}^{2}} d x\right)\right]
$$

We use the method presented in subsection 4.1 on the square $D=[0, a]^{2}$ (where $a$ is chosen such that the solution of (1.3) fits inside $D$ ).

Since the method presented in subsection 4.2 was used successfully in the study of the problem (1.1), we employ it to find the numerical solutions of (1.2). These solutions are a benchmark to which we compare the results we found using our $\Gamma$-convergence methods.

The optimal shapes obtained with the $\Gamma$-convergence method coincide with the ones found using the Fourier boundary parametrization method. The numerical results can be seen in Figure 1. To compare the accuracy of the results, we took the optimal shapes obtained with the $\Gamma$-convergence method and we isolated the 0.5 level set. We choose a point in its convex hull, the centroid $G$ of a discretization $\left\{x_{1}, \ldots, x_{l}\right\}$ of the boundary, and computed the distances from that point to the contour, denoted by $\left\{\rho_{1}, \ldots, \rho_{l}\right\}$ as well as the angles made by $G x_{i}$ with the positive $x$-axis, denoted by $\left\{\theta_{1}, \ldots, \theta_{l}\right\}$. This procedure 
gives us a radial parametrization of our domain. Using a least squares fit

$$
\min _{\left(a_{j}\right)_{j=0}^{n},\left(b_{j}\right)_{j=0}^{n}} \sum_{i=1}^{l}\left(a_{0}+\sum_{j=1}^{n} a_{j} \cos \left(j \theta_{i}\right)+\sum_{j=1}^{n} \sin \left(j \theta_{i}\right)-\rho_{i}\right)^{2}
$$

we are able to find the first $2 n+1$ Fourier coefficients of this radial function. We use these coefficients to construct the radial function of our shape $\Omega^{*}$. We use MpsPack to compute $\lambda_{k}\left(\Omega^{*}\right)+\operatorname{Per}\left(\Omega^{*}\right)$ and we compare the results, which can be seen in Table 1 . We can see that the results agree, and in general the ones obtained with the $\Gamma$-convergence method are a bit weaker, in the sense that the minimal value is higher. Still, the fact that we obtain the same shapes, with small errors, shows that the $\Gamma$-convergence method is a suitable tool for the study of problem (1.2). Furthermore, it gets close enough to the optimizer without imposing any topological constraints.

One interesting question that has been addressed in several papers ([3, 25]) is the multiplicity of $\lambda_{k}$ at the optimum. We noticed in our computations that the optimal shape for (1.3) does not always have multiple $k^{\text {th }}$ eigenvalue. This was already proved for $k=2$ in [12] and our computations have shown that for $k=6,9,13,15$ the optimal eigenvalues should be simple. This behaviour is different from the one observed for problem (1.1). It is known that if a local minimizer of problem (1.1) would have simple eigenvalue then its eigenfunction would satisfy the overdetermined problem

$$
\left\{\begin{aligned}
-\Delta u=\lambda u & \text { in } \Omega \\
u=0 & \text { on } \partial \Omega \\
\frac{\partial u}{\partial n}=c & \text { on } \partial \Omega,
\end{aligned}\right.
$$

where $c>0$ is a constant. There is a conjecture due to Bernstein [6], concerning the above overdetermined problem. The problem has been revisited in [29].

Conjecture 4.1. If problem (4.2) has a solution, then $\Omega$ is a disk.

This is true in the case of $\lambda_{1}$, but the arguments used in the proof rely essentially on the fact that the first eigenfunction can be chosen positive. In the case $k \geq 2$, the eigenfunctions corresponding to $\lambda_{k}$ are not positive, so the argument used for $k=1$ does not work here. Still, to the our knowledge, no counter-example of this conjecture is known.

A recent result by A. Berger [7] says that in two dimensions, the only positive integers $k$ for which the ball is a local minimizer for $\lambda_{k}$ under volume constraint are $k=1,3$. Thus, we have the following interesting fact:

- Suppose that for some $k \notin\{1,3\}$ the shape which $\Omega$ is solution to (1.1) has simple $k^{\text {th }}$ eigenvalue. Then the $k^{\text {th }}$ corresponding eigenfunction $u_{k}$ satisfies an overdetermined problem of the type (4.2). The result of A. Berger says that $\Omega$ cannot be a disk. Thus Conjecture 4.1 is contradicted.

Thus, Conjecture 4.1 together with the result of [7, imply that in the case of problem (1.1), if $k \geq 2$ the multiplicity is greater than one at the optimum.

In the case of the perimeter constraint, the situation is different. We can find shapes $\Omega$, which are not disks, such that the overdetermined problem

$$
\left\{\begin{aligned}
-\Delta u=\lambda u & \text { in } \Omega \\
u=0 & \text { on } \partial \Omega \\
\frac{\partial u}{\partial n}=\mathcal{H} & \text { on } \partial \Omega .
\end{aligned}\right.
$$

has a non-trivial solution. Such examples are the shape described in [12] as well as the shapes we found numerically for $k=6,9,13,15$.

We notice that the numerical optimal shape obtained for $k=3$ is the disk. This is to be expected, since it is a direct consequence of the conjecture that the disk minimizes $\lambda_{3}(\Omega)$ under volume constraint. This is still an open problem. 
We observe that all the optimal shapes computed have one or more axes of symmetry, while this is not the case for the volume constraint where the optimal shape for $k=13$ is suspected to be non-symmetric [3].

The fact that we can immediately generalize the method in three dimensions is a big advantage. One drawback is the fact that we were not able to obtain very high resolution due to the fact that the matrices involved have extremely large dimensions. The shapes presented in Figure 2 were obtained using a $40 \times 40 \times 40$ grid on $D=[0, a]^{3}$. As previously, the initial shape was concentrated around the center of the cube $D$. In the paper 12 a few conjectures were stated regarding the minimizers in higher dimensions. The first conjecture was that the optimal shape for $\lambda_{2}(\Omega)+\operatorname{Per}(\Omega)$ has cylindrical symmetry and is not convex in the three dimensional case. This can be observed in our results. To conclude that a shape is convex or not we simply apply the following procedure : we first compute a discretization of the isosurface $\{\varphi=1 / 2\}$ and estimate the exact measure of its volume (up to roundoff error). Then, in a second step we compute the convexhull of this discretized isosurface and again estimate its volume. When the volume of the convex hull is $5 \%$ greater than the volume of the original isosurface we conclude that the computed optimal profile is not convex. We have obtained non-convex shapes for $k=2,5,6,7$. Cylindrical symmetry can be observed for $k=2,3,4,5$. For $k=8$ we observe a symmetry by a rotation of angle $\pi / 2$ and for $k=10$ we observe a tetrahedral symmetry. We notice that the numerical optimal shape for $k=4$ is the approximately a ball. This is a direct consequence of the conjecture that the ball minimizes $\lambda_{4}(\Omega)$ under volume constraint in three dimensions. The optimal computed value of $\lambda_{4}$, in this case, is 255.56, while the actual eigenvalue of a ball of same surface area is approximately 253.72. We provide for each shape the value of the scale invariant expression $\lambda_{k}(\Omega) \operatorname{Per}(\Omega)$, calculated using a finite element method.

We discussed our results with P. Antunes and P. Freitas, who made computations for problem (1.2) for $k \leq 50$ in two dimensions and $k \leq 20$ in three dimensions. In two dimensions we obtained the same results (we also performed the computations up to $k=$ 501 $)$. In the three dimensional case, for $k \leq 6$ our results coincide with theirs, but for $k \geq 7$ their optimal shapes have better cost values than ours. We believe this is due to the limitation on the discretization parameter for our method in three dimensions.

We observe that in the three dimensional case, the optimal shapes we obtained numerically do not have holes. We may ask ourselves if this behaviour can be justified. In order to do this, we can analyse the, so called, topological derivative, which for a point $x \in \Omega$ and a general functional $F$ is defined as

$$
T(x)=\lim _{r \rightarrow 0} \frac{F(\Omega \backslash B(x, r))-F(\Omega)}{\varepsilon(r)},
$$

where $\varepsilon(r)$ is positive and $\varepsilon(r) \rightarrow 0$ as $r \rightarrow 0$. For more details see [22. A negative topological derivative would mean that making a small hole decreases the value of $F$. In our particular case $F(\Omega)=\lambda_{k}(\Omega)+\operatorname{Per}(\Omega)$, and for $x \in \Omega$ and $r$ small enough, we have $F(\Omega \backslash B(x, r))>F(\Omega)$, since the eigenvalue is decreasing with respect to set inclusion, and for small $r$, we have $\operatorname{Per}(\Omega \backslash B(x, r))=\operatorname{Per}(\Omega)+\operatorname{Per}(B(x, r))$. Thus, in our case, the topological derivative is always positive, and there is no interest in creating holes in order to decrease the value of the functional.

\section{Optimality CONDitions And Qualitative Results}

Once we know that a shape optimization problem has a solution, we would like to write some optimality conditions which could allow us to find further qualitative properties. An eigenvalue of the Dirichlet Laplacian associated to a shape $\Omega$ is differentiable with respect to perturbations only if it is simple. Unfortunately, solutions of (1.1) and (1.2) are conjectured

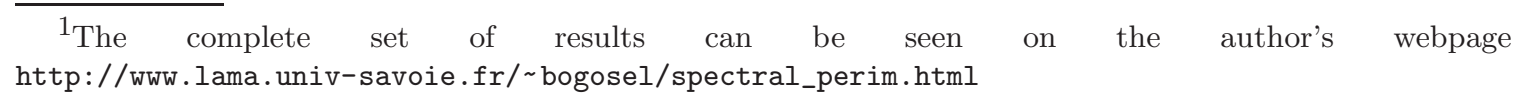



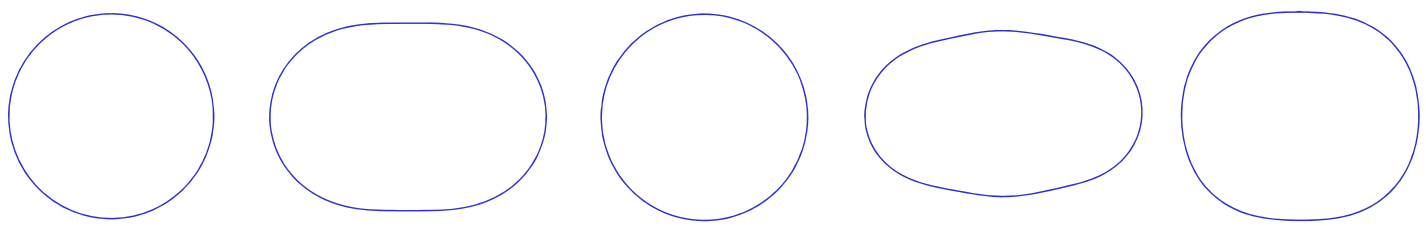

$\lambda_{1}=11.5505$

$\lambda_{2}=15.2806$

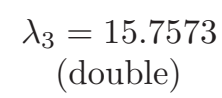

$$
\lambda_{4}=18.3496
$$$$
\lambda_{5}=19.1091
$$

(double)
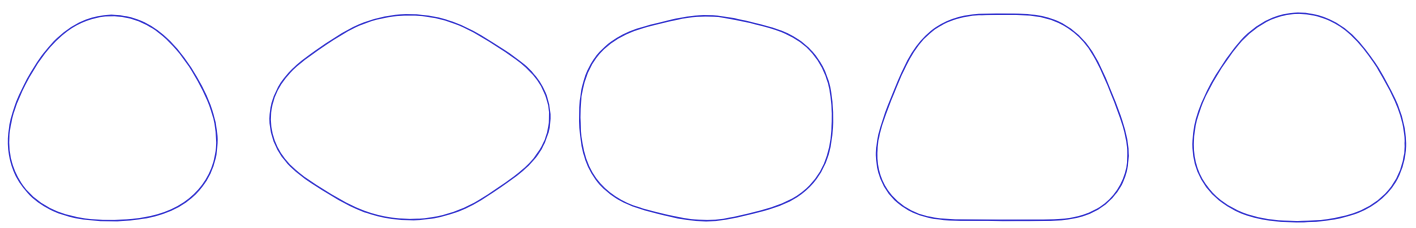

$$
\lambda_{6}=20.0909
$$

$\lambda_{7}=21.5020$

(double)

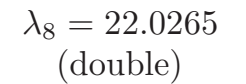

$\lambda_{9}=23.2073$

$\lambda_{10}=23.5501$

(double)
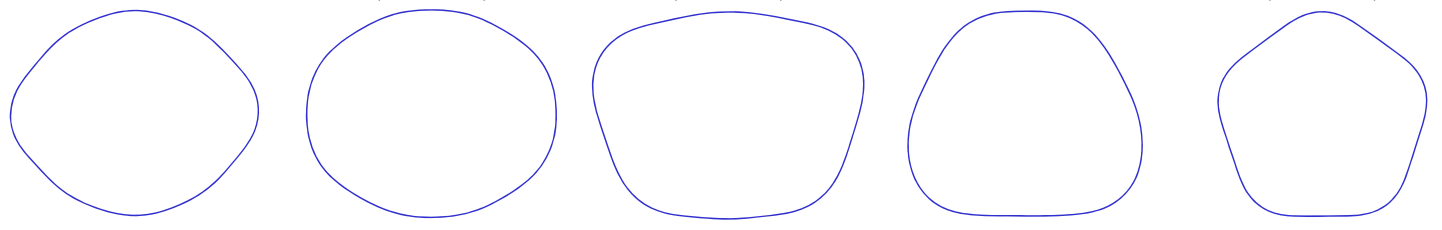

$\lambda_{11}=24.5970$

(double)

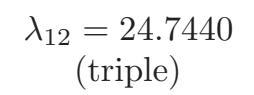

$$
\lambda_{13}=25.9823
$$

$\lambda_{14}=26.4325$

(double)

Figure 1. Numerical optimizers for problem (1.3) in 2D
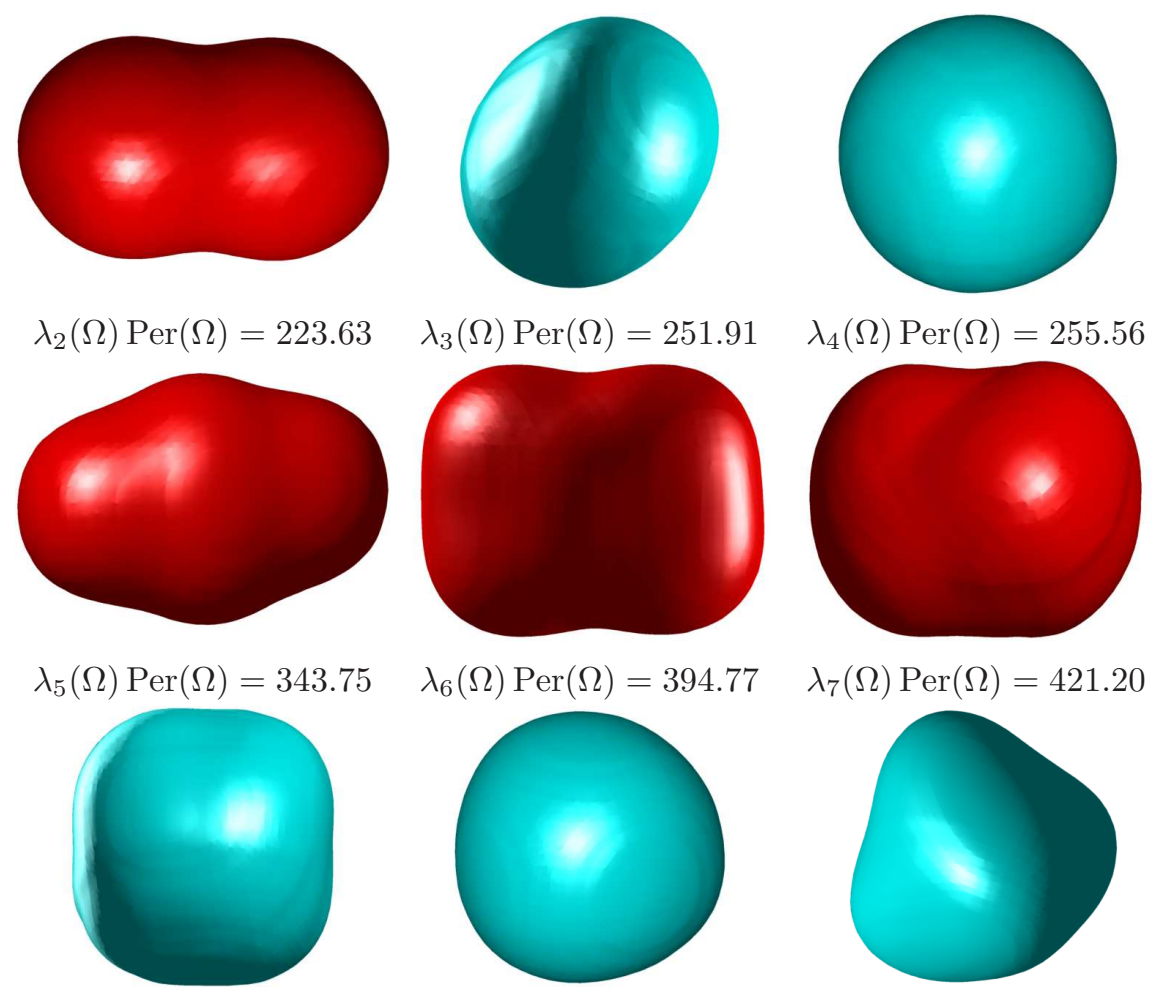

$\lambda_{8}(\Omega) \operatorname{Per}(\Omega)=439.80$

$\lambda_{9}(\Omega) \operatorname{Per}(\Omega)=446.58$

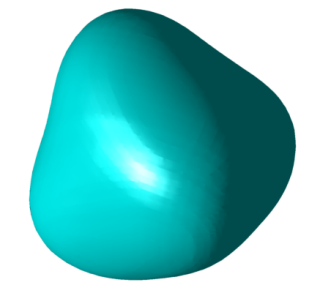

FigURE 2. Numerical optimizers for problem (1.3) in 3D Convex shapes with cyan (light); non-convex shapes with red (dark) 


\begin{tabular}{|c|c|c|c|}
\hline$k$ & mult. & $\Gamma$-conv & Fourier \\
\hline 1 & 1 & 11.55 & 11.55 \\
\hline 2 & 1 & 15.28 & 15.28 \\
\hline 3 & 2 & 15.75 & 15.75 \\
\hline 4 & 2 & 18.35 & 18.35 \\
\hline 5 & 2 & 19.11 & 19.11 \\
\hline 6 & 1 & 20.09 & 20.09 \\
\hline 7 & 2 & 21.50 & 21.50 \\
\hline 8 & 2 & 22.07 & 22.02 \\
\hline 9 & 1 & 23.21 & 23.21 \\
\hline 10 & 2 & 23.58 & 23.55 \\
\hline
\end{tabular}

\begin{tabular}{|c|c|c|c|}
\hline$k$ & mult. & $\Gamma$-conv & Fourier \\
\hline 11 & 2 & 24.62 & 24.60 \\
\hline 12 & 3 & 24.76 & 24.74 \\
\hline 13 & 1 & 25.98 & 25.98 \\
\hline 14 & 2 & 26.46 & 26.43 \\
\hline 15 & 1 & 26.91 & 26.91 \\
\hline 16 & 3 & 27.27 & 27.25 \\
\hline 17 & 3 & 27.37 & 27.36 \\
\hline 18 & 2 & 28.66 & 28.63 \\
\hline 19 & 2 & 29.09 & 29.08 \\
\hline 20 & 3 & 29.54 & 29.51 \\
\hline
\end{tabular}

TABLE 1. Comparative results

to have multiple $k^{\text {th }}$ eigenvalue at the optimum (with a few exceptions in the case of the perimeter constraint). Thus, classical optimality conditions, like the one exploited in [12, cannot be written for every $k$. In our case, we observed that for $d=2, k=2,6,9,13,15$ optimal shapes probably have simple eigenvalues. Thus we can apply the method described in 12 to deduce the fact that the boundary of these shapes does not contain any flat parts or any arcs of circles. We are led to believe that this fact should be true in the general case. To study this question in the case of multiple eigenvalues it is possible to use methods inspired by [17], [16] and [21. In the previously cited article [17, the authors provided an optimality condition for problem (1.1), which treats the case when the eigenvalue is multiple at the optimum. The results of this section are dedicated to finding a similar optimality condition for problem (1.2).

The following theorem is a result similar to Theorem 2.5.10 in [18] where it is said that if an optimizer $\Omega^{*}$ for problem (1.1) is such that the $k^{\text {th }}$ eigenvalue is multiple, then the multiplicity cluster ends at $\lambda_{k}$, i.e. $\lambda_{k}\left(\Omega^{*}\right)<\lambda_{k+1}\left(\Omega^{*}\right)$. Throughout this section we assume that $\Omega$ has boundary of class $C^{3}$. In particular, this implies that its curvature, $\mathcal{H}$ is of class $C^{1}$. This assumption is stronger than the results obtained in [15, where it is proved that the optimizer has regularity $C^{1, \alpha}$. To our knowledge, this regularity assumption cannot be easily deduced from [15], and it is an open question, though it is natural to expect it.

Theorem 5.1. Let $k \geq 2$ such that $\lambda_{k}>\lambda_{k-1}$ and assume that $\Omega$ is a minimizer for the $k^{\text {th }}$ eigenvalue of the Dirichlet Laplacian with a perimeter constraint (i.e. a solution of the problem (1.2)). Then $\lambda_{k}$ is simple and there exists a unique (up to sign) eigenfunction $u$ satisfying

$$
\begin{cases}-\Delta u=\lambda_{k}(\Omega) u & \text { in } \Omega \\ u=0 & \text { on } \partial \Omega \\ \left(\frac{\partial u}{\partial n}\right)^{2}=\mathcal{H} & \text { on } \partial \Omega\end{cases}
$$

Proof: Let $\Omega_{\varepsilon}=f_{\varepsilon}(\Omega)$ be a perimeter preserving analytic deformation of $\Omega$ and denote $\left(\Lambda_{i, \varepsilon}\right)_{i \leq p}$ and $\left(u_{i, \varepsilon}\right)_{i \leq p}$ the families of eigenfunctions and eigenvectors associated to $\lambda_{k}$ according to Lemma 2.7. We use the notation $\lambda_{k, \varepsilon}(\Omega)=\lambda_{k}\left(\Omega_{\varepsilon}\right)$. Since $\lambda_{k}=\Lambda_{i, 0}>\lambda_{k-1}$, by continuity, for sufficiently small $\varepsilon$ we have

$$
\Lambda_{i, \varepsilon}>\lambda_{k-1, \varepsilon} .
$$

We know that $\Omega$ is a local minimizer for the Dirichlet Laplacian under the considered perturbation, which means that

$$
\Lambda_{i, \varepsilon} \geq \lambda_{k, \varepsilon}
$$

The differentiable function $\varepsilon \mapsto \Lambda_{i, \varepsilon}$ achieves a local minimum at $\varepsilon=0$ and this implies $\frac{d}{d \varepsilon} \Lambda_{i, \varepsilon}=0$. 
As a consequence, the quadratic form $q_{v}$ defined in Lemma 2.8 is identically zero on $E_{k}$, where $v=\left\langle\frac{d}{d \varepsilon} f_{\varepsilon}, n\right\rangle$. The perimeter preserving deformation is arbitrary, so by Lemma 2.6 we have that $q_{v}$ vanishes on $E_{k}$ for every $v \in \mathcal{P}_{0}(\partial \Omega)$. This means that

$$
\int_{\partial \Omega}\left(\frac{\partial u}{\partial n}\right)^{2} v d \sigma=0
$$

for every $v \in \mathcal{P}_{0}(\partial \Omega)$ and for every $u \in E_{k}$.

Hence, for every function $u \in E_{k}$ there exists a constant $c>0$ such that $\left(\frac{\partial u}{\partial n}\right)^{2}=c \mathcal{H}$ on $\partial \Omega$. Since $\Omega$ is bounded, it is a classical result that the curvature of $\Omega$ is non-negative at at least one point. Thus, since $c \mathcal{H}$ is non-negative, it follows that $c \geq 0$. We cannot have $c=0$, because otherwise $\frac{\partial u}{\partial n}=0$ on $\partial \Omega$, and we could extend $u$ with $u=0$ outside $\Omega$. Thus this extension would be an eigenfunction on any set $\Omega^{\prime}$ containing $\Omega$, contradicting the uniqueness of the analytic extension.

Thus, we have $\left|\frac{\partial u}{\partial n}\right|=\sqrt{c \mathcal{H}}$ on $\partial \Omega$. Since $\mathcal{H}$ is continuous, there exists an open set $U$ such that $\mathcal{H}>0$ on $U \cap \partial \Omega$. Thus, on $\partial \Omega \cap U, \frac{\partial u}{\partial n}$ keeps constant sign, so in this set we can only have $\frac{\partial u}{\partial n}=\sqrt{c \mathcal{H}}$ or $\frac{\partial u}{\partial n}=-\sqrt{c \mathcal{H}}$. If we have two eigenfunctions $u_{1}, u_{2}$ then there exists a linear combination $u=\alpha u_{1}+\beta u_{2}$ such that $\frac{\partial u}{\partial n}$ vanishes on $\partial \Omega \cap U$. We apply Holmgren uniqueness theorem to conclude that $u=0$ and $\lambda_{k}$ is simple.

The following result connects the criticality of a domain $\Omega$ with the definiteness of the quadratic form $q_{v}$. This will allow us later to state our optimality result.

Theorem 5.2. Let $k \geq 1$ be a positive integer.

(1) If $\Omega$ is a critical domain for the $k^{\text {th }}$ eigenvalue of the Dirichlet Laplacian, then, for all $v \in \mathcal{P}_{0}(\partial \Omega)$, the quadratic form $q_{v}(u)=-\int_{\partial \Omega}\left(\frac{\partial u}{\partial n}\right)^{2} v d \sigma$ is not definite on $E_{k}$.

(2) Assume that $\lambda_{k}>\lambda_{k-1}$ or $\lambda_{k}<\lambda_{k+1}$, and that for all $v \in \mathcal{P}_{0}(\partial \Omega)$, the quadratic form $q_{v}(u)=-\int_{\partial \Omega}\left(\frac{\partial u}{\partial n}\right)^{2} v d \sigma$ is not definite on $E_{k}$. Then $\Omega$ is a critical domain for the $k^{\text {th }}$ eigenvalue of the Dirichlet Laplacian.

Proof: (1) Consider a function $v \in \mathcal{P}_{0}(\partial \Omega)$ and let $\Omega_{\varepsilon}=f_{\varepsilon}(\Omega)$ be an analytic perimeter preserving deformation of $\Omega$ such that $v=\left\langle\left.\frac{d}{d \varepsilon} f_{\varepsilon}\right|_{\varepsilon=0}, n\right\rangle$ (such a deformation exists by Lemma 2.6). Let $\left(\Lambda_{i, \varepsilon}\right)_{i \leq p}$ and $\left(u_{i, \varepsilon}\right)_{i \leq p}$ be families of eigenvalues and eigenfunctions associated to $\lambda_{k}$ like in Lemma 2.7. There exist two integers $i, j \leq p$ such that $\left.\frac{d}{d \varepsilon} \lambda_{k, \varepsilon}\right|_{\varepsilon=0^{-}}=\left.\frac{d}{d \varepsilon} \Lambda_{i, \varepsilon}\right|_{\varepsilon=0}$ and $\left.\frac{d}{d \varepsilon} \lambda_{k, \varepsilon}\right|_{\varepsilon=0^{+}}=\left.\frac{d}{d \varepsilon} \Lambda_{j, \varepsilon}\right|_{\varepsilon=0}$. The criticality of $\Omega$ implies that $\left.\frac{d}{d \varepsilon} \Lambda_{i, \varepsilon}\right|_{\varepsilon=0} \times\left.\frac{d}{d \varepsilon} \Lambda_{j, \varepsilon}\right|_{\varepsilon=0} \leq 0$ and from Lemma 2.8 , it follows that $q_{v}$ has both positive and negative eigenvalues, which means that $q_{v}$ is not definite on $E_{k}$.

(2) Assume $\lambda_{k}>\lambda_{k-1}$ and let $\Omega_{\varepsilon}=f_{\varepsilon}(\Omega)$ be a perimeter-preserving deformation of $\Omega$. Let $\left(\Lambda_{i, \varepsilon}\right)_{i \leq p}$ and $\left(u_{i, \varepsilon}\right)_{i \leq p}$ be families of eigenvalues and eigenfunctions associated to $\lambda_{k}$ according to Lemma 2.7. For $\varepsilon$ sufficiently small we have $\lambda_{k, \varepsilon}=\min _{i \leq p} \Lambda_{i, \varepsilon}$. Hence

$$
\left.\frac{d}{d \varepsilon} \lambda_{k, \varepsilon}\right|_{\varepsilon=0^{+}}=\left.\min _{i \leq p} \frac{d}{d \varepsilon} \Lambda_{i, \varepsilon}\right|_{\varepsilon=0}
$$

and

$$
\left.\frac{d}{d \varepsilon} \lambda_{k, \varepsilon}\right|_{\varepsilon=0^{-}}=\left.\max _{i \leq p} \frac{d}{d \varepsilon} \Lambda_{i, \varepsilon}\right|_{\varepsilon=0} .
$$

The non definiteness of $q_{v}$ on $E_{k}$ means that its smallest eigenvallue is non positive and its largest one is non negative. This implies that

$$
\left.\frac{d}{d \varepsilon} \lambda_{k, \varepsilon}\right|_{\varepsilon=0^{+}}=\left.\min _{i \leq p} \frac{d}{d \varepsilon} \Lambda_{i, \varepsilon}\right|_{\varepsilon=0} \leq 0
$$

and

$$
\left.\frac{d}{d \varepsilon} \lambda_{k, \varepsilon}\right|_{\varepsilon=0^{-}}=\left.\max _{i \leq p} \frac{d}{d \varepsilon} \Lambda_{i, \varepsilon}\right|_{\varepsilon=0} \geq 0
$$


which in turn implies the criticality of the domain $\Omega$.

The case $\lambda_{k}<\lambda_{k+1}$ can be treated in a similar manner.

The next result provides a nice characterisation of the non-definitness of $q_{v}$. Note that unlike in [17, we have to add a hypothesis on $\mathcal{H}$. This hypothesis is natural when dealing with solutions of problem (1.2).(see [15], Section 4.)

Theorem 5.3. Let $k$ be a natural integer. If $\Omega$ is bounded and its curvature satisfies $\mathcal{H} \geq 0$ then the following two conditions are equivalent:

(i) For all $v \in \mathcal{P}_{0}(\partial \Omega)$, the quadratic form $q_{v}$ is not definite on $E_{k}$.

(ii) There exists a finite family of eigenfunctions $\left(u_{i}\right)_{i \leq m} \subset E_{k}$ satisfying

$$
\sum_{i=1}^{m}\left(\frac{\partial u_{i}}{\partial n}\right)^{2}=\mathcal{H} \text { on } \partial \Omega
$$

Proof: To see that (ii) implies (i) it suffices to notice that, for any $v \in \mathcal{P}_{0}(\partial \Omega)$

$$
\sum_{i \leq m} q_{v}\left(u_{i}\right)=-\sum_{i \leq m} \int_{\partial \Omega}\left(\frac{\partial u_{i}}{\partial n}\right)^{2} v d \sigma=-\int_{\partial \Omega} \mathcal{H} v d \sigma=0,
$$

which means that $q_{v}$ is not definite on $E_{k}$.

To prove the other implication we look at $K=\operatorname{conv}\left\{\left(\frac{\partial u}{\partial n}\right)^{2}, u \in E_{k}\right\}$, and we want to prove that the function $\mathcal{H}$ belongs to $K$. Suppose that $\mathcal{H} \notin K$. Then, from the Hahn-Banach theorem (applied to the finite dimensional normed vector subspace of $C^{1}(\partial \Omega)$ spanned by $K$ and $\mathcal{H})$, there exists a function $v \in C^{1}(\partial \Omega)$ such that $\int_{\partial \Omega} \mathcal{H} v d \sigma>0$ and for all $u \in E_{k}$,

$$
\int_{\partial \Omega}\left(\frac{\partial u}{\partial n}\right)^{2} v d \sigma \leq 0
$$

Since $v$ is not necessarily in $\mathcal{P}_{0}(\partial \Omega)$, we modify it by a constant term and define $v_{0}=v-c$ where $c$ is chosen such that $v_{0} \in \mathcal{P}_{0}(\partial \Omega)$. The condition that $c$ must satisfy is

$$
0=\int_{\partial \Omega} \mathcal{H} v_{0} d \sigma=\int_{\partial \Omega} \mathcal{H} v d \sigma-c \int_{\partial \Omega} \mathcal{H} d \sigma
$$

This last relation defines $c=\frac{\int_{\partial \Omega} \mathcal{H} v d \sigma}{\int_{\partial \Omega} \mathcal{H} d \sigma}$, since $\int_{\partial \Omega} \mathcal{H} d \sigma>0$. The fact that $\int_{\partial \Omega} \mathcal{H} d \sigma>0$ is a consequence of the fact that $\mathcal{H} \geq 0$ and $\Omega$ is bounded. With the above considerations, we see that $c>0$.

For $u \in E_{k}$ we have

$$
\begin{aligned}
q_{v_{0}}(u) & =-\int_{\partial \Omega}\left(\frac{\partial u}{\partial n}\right)^{2} v_{0} d \sigma \\
& =-\int_{\partial \Omega}\left(\frac{\partial u}{\partial n}\right)^{2} v d \sigma+c \int_{\partial \Omega}\left(\frac{\partial u}{\partial n}\right)^{2} d \sigma \\
& \geq c \int_{\partial \Omega}\left(\frac{\partial u}{\partial n}\right)^{2} d \sigma
\end{aligned}
$$

and $\int_{\partial \Omega}\left(\frac{\partial u}{\partial n}\right)^{2} d \sigma>0$ for any non trivial Dirichlet eigenfunction $u$ (due to Holmgren uniqueness theorem). In conclusion, we have found a function $v_{0} \in \mathcal{P}_{0}(\partial \Omega)$ such that the quadratic form $q_{v_{0}}$ is positive definite on $E_{k}$, which contradicts condition (i).

Corollary 5.4. If $\Omega$ is a local minimizer for the problem (1.2)

$$
\min _{\operatorname{Per}(\Omega)=1} \lambda_{k}(\Omega)
$$


with boundary of class $C^{3}$, then there exists a finite family of eigenfunctions $\left(u_{i}\right)_{i \leq m} \subset E_{k}$, such that

$$
\sum_{i=1}^{m}\left(\frac{\partial u_{i}}{\partial n}\right)^{2}=\mathcal{H} .
$$

Proof: It is a direct result of the above theorems, noting that any solution $\Omega$ of the problem must verify $\mathcal{H} \geq 0$ [15].

Remark 5.5. We note that Corollary 5.4 the number $m$ of eigenfunctions that satisfy the optimality condition is not known. Numerical computations done in Section 6 suggest that $m$ is equal to the multiplicity of $\lambda_{k}$.

Once this regularity result is established, we can apply the bootstrap procedure presented in [12], and conclude that $\Omega$ is smooth.

Corollary 5.6. If $\Omega$ is a minimizer for the problem (1.2), with boundary of class $C^{3}$, then $\Omega$ has boundary of class $C^{\infty}$.

Proof: If $\Omega$ is of class $C^{3}$, then Corollary 5.4 holds and we have the optimality relation

$$
\sum_{i=1}^{m}\left(\frac{\partial u_{i}}{\partial n}\right)^{2}=\mathcal{H} .
$$

Since $\Omega$ is of class $C^{2, \alpha}$, it follows, using standard Shauder regularity estimates, that $\partial_{n} u_{i}^{2}$ is $C^{1, \alpha}$. The optimality relation, then implies that $\mathcal{H}$ is $C^{1, \alpha}$, and thus $\Omega$ is of class $C^{3, \alpha}$. Iterating this procedure we find that $\Omega$ is of class $C^{\infty}$.

In the article 12 the authors prove that the solution of (1.2) in the case $k=2, d=2$ has no segments and no arcs of circles in its boundary. The method used in the mentioned article works only in the case we know the corresponding eigenvalue is simple. Using the above corollary, we can partially extend this result to the general case. In the following, we call a flat part of $\mathbb{R}^{d}$, the nonempty intersection of a $d-1$ dimensional hyperplane with a $d$-dimensional open ball.

Theorem 5.7. If $\Omega$ is a local minimizer for the problem 1.2

$$
\min _{\operatorname{Per}(\Omega)=1} \lambda_{k}(\Omega)
$$

then $\partial \Omega$ does not contain a flat parts.

Proof: Suppose that $\Omega$ contains a flat part $S$ in its boundary. Using the previous convention, $S=H \cap B$ where $H$ is a $d-1$ dimensional hyperplane and $B$ is a $d$-dimensional ball. Then $\mathcal{H}=0$ on that region $S$, and by Corollary [5.4, at least one eigenfunction $u$ satisfies $\frac{\partial u}{\partial n}=0$ on that $S$.

We then choose an extension $\Omega^{\prime}=\Omega \cup B^{\prime}$ of the domain $\Omega$ such that $B^{\prime}$ is a ball, $B^{\prime} \subset B$, $B^{\prime} \not \subset \Omega$ and $B^{\prime}$ is small enough such that $B^{\prime} \cap \partial \Omega \subset S$. Define $u^{\prime}=u$ on $\Omega$ and 0 on $\Omega^{\prime} \backslash \Omega$. In this way, we create an eigenfunction $u^{\prime}$ on $\Omega^{\prime}$ which is zero on an open set. This together with the analiticity of $u^{\prime}$ and the fact that $u^{\prime}$ is not identically zero brings us to a contradiction.

In conclusion, $\Omega$ cannot contain a flat part in its boundary.

\section{NumericAl OBSERVATION OF THE OPTIMALITY CONDITIONS}

By the above results, we know that if $\Omega$ is a minimizer for (1.2) then it exists a family of eigenfunctions $\left(u_{i}\right)_{i=1}^{m} \subset E_{k}$ such that

$$
\sum_{i=1}^{m}\left(\frac{\partial u_{i}}{\partial n}\right)^{2}=\mathcal{H}
$$


In order to evaluate the numerical quality of our solutions we would like to investigate how far our solutions satisfy this optimality condition. The question is whether we are able to find a combination of eigenfunctions which realize this equality. Suppose that $\operatorname{dim} E_{k}=p$ and the $p$ orthonormal eigenfunctions which span $E_{k}$ are denoted $\mathfrak{u}_{1}, \ldots, \mathfrak{u}_{p}$. It is easy to see that (6.1) implies that

$$
\mathcal{H} \in \operatorname{span}\left(\left\{\left(\frac{\partial \mathfrak{u}_{i}}{\partial n}\right)^{2}, i=1 . . p\right\} \bigcup\left\{\frac{\partial \mathfrak{u}_{i}}{\partial n} \frac{\partial \mathfrak{u}_{j}}{\partial n}, 1 \leq i<j \leq p\right\}\right) .
$$

This observation is a direct consequence of the fact that each $u_{i}$ can be written as

$$
u_{i}=\sum_{j=1}^{p} \alpha_{j}^{i} \mathfrak{u}_{j}
$$

Thus, in a first step, we can find the coefficients of $\mathcal{H}$ in the decomposition

$$
\mathcal{H}=\sum_{i=1}^{p} \alpha_{i}\left(\frac{\partial \mathfrak{u}_{i}}{\partial n}\right)^{2}+\sum_{1 \leq i<j \leq p} \beta_{i, j} \frac{\partial \mathfrak{u}_{i}}{\partial n} \frac{\partial \mathfrak{u}_{j}}{\partial n}
$$

by solving an optimization problem. The normal derivatives $\frac{\partial \mathfrak{u}_{i}}{\partial n}$ and the curvature are known on a discretization $\left\{x_{1}, \ldots, x_{l}\right\}$ of the boundary $\partial \Omega$. To find the coefficients, we solve the quadratic, convex minimization problem

$$
\min _{\substack{\left(\alpha_{i}\right)_{i=1}^{p},\left(\beta_{i, j}\right)_{1 \leq i<j \leq n}}} \sum_{h=1}^{l}\left(\sum_{i=1}^{p} \alpha_{i}\left(\frac{\partial \mathfrak{u}_{i}}{\partial n}\left(x_{h}\right)\right)^{2}+\sum_{1 \leq i<j \leq p} \beta_{i, j} \frac{\partial \mathfrak{u}_{i}}{\partial n}\left(x_{h}\right) \frac{\partial \mathfrak{u}_{j}}{\partial n}\left(x_{h}\right)-\mathcal{H}\left(x_{h}\right)\right)^{2}
$$

Then, we transform this quadratic representation into a canonical representation by using the classical Gauss-Jacobi method. Of course, this representation is not unique. The claim of Corollary 5.4 is that this canonical representation consists of a sum of squares. In order to test this we check if the matrix $\left(a_{i, j}\right)$ defined by $a_{i, i}=\alpha_{i}, a_{i, j}=a_{j, i}=\beta_{i, j} / 2$ is positive definite. The answer is affirmative and a representation of the type (6.1) can be found for each of the numerical optimal shapes. For $k \leq 15$ the numerical optimality relation is satisfied with a pointwise error smaller than $10^{-4}$.

We present below a few other numerical observations in connection with the optimality conditions.

- If $x \in \partial \Omega$ and $\mathcal{H}(x)=0$, then all nodal lines corresponding to the eigenfunctions present in the optimality relation touch $\partial \Omega$ at $x$. This is observed numerically in Figure 3 for $k=18$.

- Numerical observations suggest that the number of eigenfunctions $m$ present in the optimality condition is equal to the multiplicity of the eigenvalue at the optimum. Furthermore, the relation seems to be a convex combination of the type

$$
a_{k}\left(\partial_{n} \mathfrak{u}_{k}\right)^{2}+a_{k-1}\left(\partial_{n} \mathfrak{u}_{k-1}\right)^{2}+\ldots+a_{k-m+1}\left(\partial_{n} \mathfrak{u}_{k-m+1}\right)^{2}=\mathcal{H},
$$

where $a_{k}+a_{k-1}+\ldots+a_{k-m+1}=1$.

- Motivated by [24], we studied numerically some convex combinations of eigenvalues under perimeter constraint. Suppose that $\Omega^{*}$ is solution of problem (1.2) and $\lambda_{k}\left(\Omega^{*}\right)$ is double, with observed numerical optimality relation

$$
a\left(\partial_{n} \mathfrak{u}_{k}\right)^{2}+(1-a)\left(\partial_{n} \mathfrak{u}_{k-1}\right)^{2}=\mathcal{H} .
$$

Then, we observed numerically that $\Omega^{*}$ is also a solution of the problem

$$
\min _{\operatorname{Per}(\Omega)=1}\left(\alpha \lambda_{k}(\Omega)+(1-\alpha) \lambda_{k-1}(\Omega)\right),
$$

for every $\alpha \in[a, 1]$. This can be generalized to the cases where the eigenvalue has higher multiplicity. 

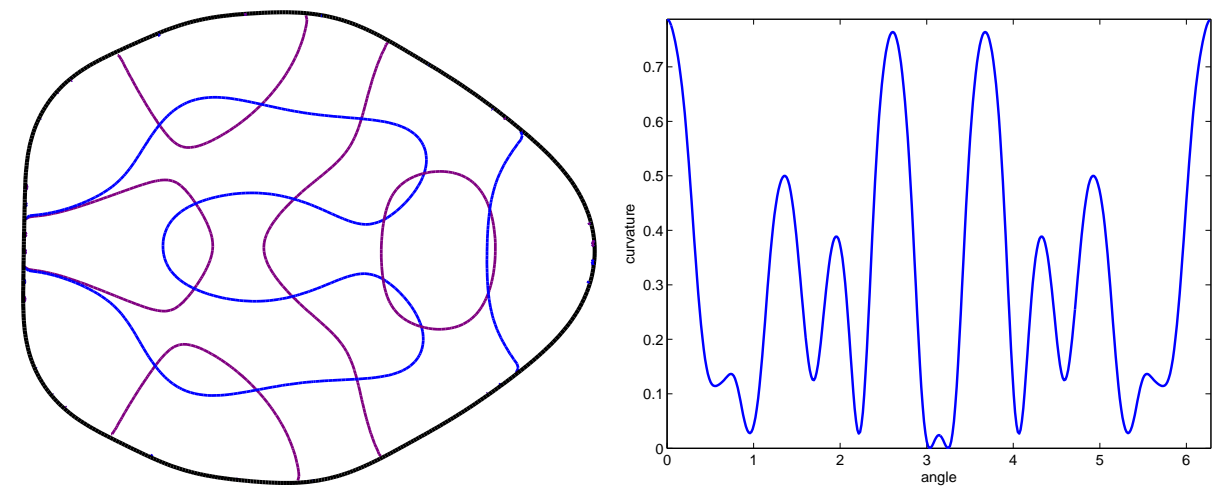

Figure 3 . Optimal set $\Omega_{18}$ obtained for $k=18$, together with the nodal lines of the eigenfunctions $u_{17}, u_{18}$ corresponding to $\lambda_{17}\left(\Omega_{18}\right)=\lambda_{18}\left(\Omega_{18}\right)$.

(left) Plot of the curvature of $\Omega_{18}$; note that the nodal lines touch the boundary at the two points having zero curvature. (right)

\begin{tabular}{|c|c|c|c|c|}
\hline$k$ & ault. & Numerical optimality relation & $L^{2}$ error & $L^{\infty}$ error \\
\hline 1 & 1 & $\left(\frac{\partial u_{1}}{\partial n}\right)^{2}=\mathcal{H}$ & 0 & $\overline{0}$ \\
\hline 2 & 1 & $\left(\frac{\partial u_{2}}{\partial n}\right)^{2}=\mathcal{H}$ & $3 \cdot 10^{-}$ & $4 \cdot 10^{-8}$ \\
\hline 3 & 2 & $\left(\frac{1}{\sqrt{2}} \frac{\partial u_{2}}{\partial n}\right)^{2}+\left(\frac{1}{\sqrt{2}} \frac{\partial u_{3}}{\partial n}\right)^{2}=\mathcal{H}$ & $5 \cdot 10^{-4}$ & $7 \cdot 10^{-4}$ \\
\hline 4 & 2 & $\left(0.16 \frac{\partial u_{3}}{\partial n}\right)^{2}+\left(0.98 \frac{\partial u_{4}}{\partial n}\right)^{2}=\mathcal{H}$ & $3 \cdot 10^{-4}$ & $6 \cdot 10^{-4}$ \\
\hline 5 & 2 & $\left(0.54 \frac{\partial u_{4}}{\partial n}\right)^{2}+\left(0.84 \frac{\partial u_{5}}{\partial n}\right)^{2}=\mathcal{H}$ & $2 \cdot 10^{-4}$ & $3 \cdot 10^{-4}$ \\
\hline 6 & 1 & $\left(\frac{\partial u_{6}}{\partial n}\right)^{2}=\mathcal{H}$ & $3 \cdot 10^{-4}$ & $5 \cdot 10^{-4}$ \\
\hline 7 & 2 & $\left(0.87 \frac{\partial u_{6}}{\partial n}\right)^{2}+\left(0.48 \frac{\partial u_{7}}{\partial n}\right)^{2}=\mathcal{H}$ & $4 \cdot 10^{-4}$ & $5 \cdot 10^{-4}$ \\
\hline 8 & 2 & $\left(0.39 \frac{\partial u_{7}}{\partial n}\right)^{2}+\left(0.92 \frac{\partial u_{8}}{\partial n}\right)^{2}=\mathcal{H}$ & $3 \cdot 10^{-4}$ & $3 \cdot 10^{-4}$ \\
\hline 9 & 1 & $\left(\frac{\partial u_{9}}{\partial n}\right)^{2}=\mathcal{H}$ & $7 \cdot 10^{-5}$ & $10^{-4}$ \\
\hline 10 & 2 & $\left(\frac{1}{\sqrt{2}} \frac{\partial u_{9}}{\partial n}\right)^{2}+\left(\frac{1}{\sqrt{2}} \frac{\partial u_{10}}{\partial n}\right)^{2}=\mathcal{H}$ & $2 \cdot 10^{-4}$ & $2 \cdot 10^{-4}$ \\
\hline 11 & 2 & $\left(0.51 \frac{\partial u_{10}}{\partial n}\right)^{2}+\left(0.86 \frac{\partial u_{11}}{\partial n}\right)^{2}=\mathcal{H}$ & $6 \cdot 10^{-4}$ & $7 \cdot 10^{-4}$ \\
\hline 12 & 3 & $\begin{array}{c}\left(0.31 \frac{\partial u_{10}}{\partial n}\right)^{2}+\left(0.51 \frac{\partial u_{11}}{\partial n}\right)^{2}+ \\
\left(0.80 \frac{\partial u_{12}}{\partial n}\right)^{2}=\mathcal{H}\end{array}$ & $3 \cdot 10^{-5}$ & $2 \cdot 10^{-5}$ \\
\hline 13 & 1 & $\left(\frac{\partial u_{13}}{\partial n}\right)^{2}=\mathcal{H}$ & $4 \cdot 10^{-4}$ & $5 \cdot 10^{-4}$ \\
\hline 14 & 2 & $\left(0.82 \frac{\partial u_{13}}{\partial n}\right)^{2}+\left(0.57 \frac{\partial u_{14}}{\partial n}\right)^{2}=\mathcal{H}$ & $2 \cdot 10^{-5}$ & $2 \cdot 10^{-5}$ \\
\hline 15 & 1 & $\left(\frac{\partial u_{15}}{\partial n}\right)^{2}=\mathcal{H}$ & $10^{-5}$ & $10^{-5}$ \\
\hline
\end{tabular}

TABLE 2. Numerical optimality conditions in dimension two

\section{ACKNOWLEDGEMENTS}

We wish to thank Dorin Bucur for the stimulating discussions we had with him on the topics presented in this article. The authors thank Pedro Antunes and Pedro Freitas for sharing their results, obtained independently while working on the same problem. The authors thank the anonymous reviewers for their suggestions and remarks which helped improve the quality of the paper.

\section{REFERENCES}

[1] G. Alberti. Variational models for phase transitions, an approach via gamma-convergence. 1998.

[2] L. Ambrosio, N. Fusco, and D. Pallara. Functions of bounded variation and free discontinuity problems. Oxford Mathematical Monographs. The Clarendon Press, Oxford University Press, New York, 2000.

[3] P. R. S. Antunes and P. Freitas. Numerical optimization of low eigenvalues of the Dirichlet and Neumann Laplacians. J. Optim. Theory Appl., 154(1):235-257, 2012. 
[4] P. R. S. Antunes and P. Freitas. Optimisation of eigenvalues of the Dirichlet Laplacian with a surface area restriction. 2014.

[5] A. H. Barnett and T. Betcke. Stability and convergence of the method of fundamental solutions for Helmholtz problems on analytic domains. J. Comput. Phys., 227(14):7003-7026, 2008.

[6] C. A. Berenstein. An inverse spectral theorem and its relation to the Pompeiu problem. J. Analyse Math., 37:128-144, 1980.

[7] A. Berger. The eigenvalues of the Laplacian with Dirichlet boundary condition in $\mathbb{R}^{2}$ are almost never minimized by disks. Ann. Global Anal. Geom., 47(3):285-304, 2015.

[8] B. Bourdin, D. Bucur, and É. Oudet. Optimal partitions for eigenvalues. SIAM J. Sci. Comput., 31(6):4100-4114, 2009/10.

[9] A. Braides. Approximation of Free-Discontinuity Problems. Springer, 1998.

[10] A. Braides. Gamma-Convergence for beginners. Oxford University Press, 2002.

[11] D. Bucur and G. Buttazzo. Variational methods in shape optimization problems. Progress in Nonlinear Differential Equations and their Applications, 65. Birkhäuser Boston, Inc., Boston, MA, 2005.

[12] D. Bucur, G. Buttazzo, and A. Henrot. Minimization of $\lambda_{2}(\Omega)$ with a perimeter constraint. Indiana Univ. Math. J., 58(6):2709-2728, 2009.

[13] G. Buttazzo. Gamma-convergence and its Applications to Some Problems in the Calculus of Variations. School on Homogenization ICTP, Trieste, September 6-17, 1993.

[14] G. Dal Maso and F. Murat. Asymptotic behaviour and correctors for Dirichlet problems in perforated domains with homogeneous monotone operators. Ann. Scuola Norm. Sup. Pisa Cl. Sci. (4), 24(2):239290, 1997.

[15] G. De Philippis and B. Velichkov. Existence and regularity of minimizers for some spectral functionals with perimeter constraint. Appl. Math. Optim., 69(2):199-231, 2014.

[16] A. El Soufi and S. Ilias. Riemannian manifolds admitting isometric immersions by their first eigenfunctions. Pacific J. Math., 195(1):91-99, 2000.

[17] A. El Soufi and S. Ilias. Domain deformations and eigenvalues of the Dirichlet Laplacian in a Riemannian manifold. Illinois J. Math., 51(2):645-666 (electronic), 2007.

[18] A. Henrot. Extremum problems for eigenvalues of elliptic operators. Frontiers in Mathematics. Birkhäuser Verlag, Basel, 2006.

[19] A. Henrot and M. Pierre. Variation et optimisation de formes, volume 48 of Mathématiques 8 Applications (Berlin) [Mathematics 83 Applications]. Springer, Berlin, 2005. Une analyse géométrique. [A geometric analysis].

[20] L. Modica and S. Mortola. Un esempio di $\Gamma^{-}$-convergenza. Boll. Un. Mat. Ital. B (5), 14(1):285-299, 1977.

[21] N. Nadirashvili. Berger's isoperimetric problem and minimal immersions of surfaces. Geom. Funct. Anal., 6(5):877-897, 1996.

[22] A. A. Novotny and J. Sokołowski. Topological derivatives in shape optimization. Interaction of Mechanics and Mathematics. Springer, Heidelberg, 2013.

[23] B. Osting. Optimization of spectral functions of Dirichlet-Laplacian eigenvalues. J. Comput. Phys., $229(22): 8578-8590,2010$.

[24] B. Osting and C.-Y. Kao. Minimal convex combinations of three sequential Laplace-Dirichlet eigenvalues. Appl. Math. Optim., 69(1):123-139, 2014.

[25] É. Oudet. Numerical minimization of eigenmodes of a membrane with respect to the domain. ESAIM Control Optim. Calc. Var., 10(3):315-330 (electronic), 2004.

[26] É. Oudet. Approximation of partitions of least perimeter by $\Gamma$-convergence: around Kelvin's conjecture. Exp. Math., 20(3):260-270, 2011.

[27] M. Schmidt. minfunc, 2012. http://www.cs.ubc.ca/ schmidtm/Software/minFunc.html.

[28] L. Stewart. Matlab lbfgs wrapper. http://www.cs.toronto.edu/ liam/software.shtml.

[29] S. A. Williams. Boundary regularity for a family of overdetermined problems for the Helmholtz equation. J. Math. Anal. Appl., 274(1):296-304, 2002. 